\title{
The ups and downs of space and time: Topography in Yupno language, culture, and cognition
}

\author{
Kensy Cooperrider ${ }^{1}$, James Slotta ${ }^{2}$, Rafael Núñez ${ }^{3}$ \\ ${ }^{1}$ University of Chicago, Department of Psychology \\ ${ }^{2}$ University of Texas at Austin, Department of Anthropology \\ ${ }^{3}$ University of California - San Diego, Department of Cognitive Science
}

\begin{abstract}
Much prior research has investigated how humans understand time using body-based contrasts like front/back and left/right. It has recently come to light, however, that some communities instead understand time using environment-based contrasts. Here, we present the richest portrait yet of one such case: the topographic system used by the Yupno of Papua New Guinea, in which the past is construed as downhill and the future as uphill. We first survey topographic concepts in Yupno language and culture, showing how they constitute a privileged resource for communicating about space. Next, we survey time concepts in Yupno, focusing on how topographic concepts are used to construe past, present, and future. We then illustrate how this topographic understanding of time comes to life in the words, hands, and minds of Yupno speakers. Drawing on informal interviews, we offer a view of the topographic system that goes beyond a community-level summary, and offers a glimpse of its individual-level and moment-tomoment texture. Finally, we step back to account for how this topographic understanding of time is embedded within a rich cognitive ecology of linguistic, cultural, gestural, and architectural practices. We close by discussing an elusive question: Why is the future uphill?
\end{abstract}

keywords: time; space; abstract concepts; gesture; cognitive ecology

Accepted for publication in Language and Cognition

Note: This manuscript has not undergone final copy-editing and may differ from the final published version. 


\section{Introduction}

Time is elusive. Nobody has ever seen tomorrow or touched yesterday; philosophers have been squabbling for more than a century over the nature of now. And yet time is a foundational part of human experience. Countless everyday activities - from farming and fermenting, to keeping records and conducting rituals - depend on our ability to track and compare durations, to remember and relate sequences, to reconstruct the past and plan for the future. Augustine of Hippo noted this double aspect of time already around 400 C.E.: "Who can even form a conception of it to be put in words? Yet what do we mention more often or familiarly in our conversation than time?" (Saint Augustine, ca. 400 C.E./2008, 267). Faced with this tension, humans around the world have converged on a solution: we make sense of time by construing it in terms of space, a more tangible dimension of experience (e.g., Bender \& Beller, 2014; Boroditsky, 2000; Casasanto \& Boroditsky, 2008; Clark, 1973; Lakoff \& Johnson, 1980; Moore, 2014; Núñez \& Sweetser, 2006; Traugott, 1978; Whorf, 1956). To express the passing of time, we use concepts of movement; to compare different durations we invoke length or size; to construe sequences of events — or to distinguish past from future-we repurpose ideas about location (Núñez \& Cooperrider, 2013). In this way, we are able to form conceptions of time and put those conceptions into words.

Most of the spatial construals of time studied to date have involved body-based spatial contrasts. Notions of bodily 'front' and 'back,' for instance, are widely used to characterize the position of events in a sequence-what is often called sequence time (e.g., Núñez \& Cooperrider, 2013). The construal is apparently based on the imagined scenario of entities in a line all moving in the same direction, like people walking on a path (e.g., Moore, 2014). An entity in front of 
another arrives before it (related to the use of "before" to mean 'in front of'); an entity behind another arrives after it (related to the use of "aft" to mean 'toward the back').

Also widespread is the use of the front-back contrast to distinguish past, present, and future — what is often called deictic time (e.g., Núñez \& Cooperrider, 2013). In the most common construal, evident in English, the future is what lies "ahead" of us, something we "look forward to"; the past is what we leave "behind" and "think back on." Such a future-in-front construal is found beyond English and European languages - in Mandarin, Japanese, and Wolof, for example (Alverson, 1994; Gu et al., 2019; Moore, 2014)—and appears to be rooted in the experience of forward motion: when we walk, things ahead of us in space are also further into the future; things behind us are also in the past. Commonplace and intuitive as this mapping may be, it is not used everywhere. The Aymara of the Andes construe the past as 'in front' of them and the future as 'behind,' a "reversed" mapping likely based on the idea that past is visible and known — much like what is physically in front of us - whereas the future is not yet visible and remains unknown—much like what is at our backs (Núñez \& Sweetser, 2006).

Body-based construals of time are especially explicit when they pop up in language, but they also show up in psycholinguistic experiments (e.g., Bergen \& Lau, 2012; Rinaldi et al., 2016; Walker et al., 2017) and in the gestures people spontaneously produce when they speak (e.g., Cooperrider \& Núñez, 2009; Núñez \& Sweetser, 2006). These more implicit sources of evidence reveal that people use more than just the front-back bodily axis to conceptualize time. Speakers of English and European languages also construe time in terms of their left-right axis (e.g., Santiago et al., 2007), with earlier events toward their left and later events toward their right. In fact, studies find that, when people in these groups gesture about time, they use their left-right axis more than their front-back axis (Casasanto \& Jasmin, 2012; Valenzuela et al., 
2020; Walker \& Cooperrider, 2016). This later-is-rightward construal appears to be based on experience with graphical representations such as calendars, timelines, and text generally, an interpretation supported by findings from other graphical traditions (e.g., Fuhrman \& Boroditsky, 2010; Starr \& Srinivasan, 2021). Taken together, such observations reveal a widespread tendency for humans to construe time in terms of their own bodily axes, whether front-back, leftright, or up-down. Such construals emerge overtly in language as well as in more implicit behaviors, and are rooted in basic experiences of walking, seeing, and interacting with graphical representations.

Widespread as these patterns are, they are not universal. Work in the past decade has now documented communities that construe time according to environment-based rather than bodybased contrasts (Boroditsky \& Gaby, 2010; Gaby, 2012; Núñez et al., 2012). In our own prior work, we showed that the Yupno people of Papua New Guinea tend to construe the past as downhill and the future as uphill (Núñez et al., 2012). More recently, additional reports have surfaced from the same region of Papua New Guinea, suggesting the future-uphill construal might be an areal feature (Pennington, 2016; Sarvasy, 2014; Taylor, 2015). A similar futureuphill pattern has also been documented in Tzeltal (Brown, 2012), and there are further hints of it elsewhere (Forker, 2020). Together these observations suggest that environment-based construals of time, while only glancingly described to date, are a recurring feature of the human conceptual landscape.

The use of environment-based construals of time in the Yupno and other groups is no accident: it appears to build on a preference for environment-based construals of space. Over the last three decades, abundant evidence has accumulated that—contra the long-held assumptions of Western philosophers and cognitive scientists (see Levinson \& Brown, 1994)—not all cultures 
prefer to parse the world in terms of 'front' and 'back,' 'left' and 'right'. In many communities, people instead talk about motion, location, and orientation primarily in terms of contrasts like 'east' and 'west' (e.g., Haun et al., 2011; Haviland, 1998), 'uphill' and 'downhill' (e.g., Bickel, 1997; Brown \& Levinson, 1993), 'toward the mountain' and 'toward the sea' (e.g., Wassmann \& Dasen, 1998); or in terms of wind (e.g., Hoffmann, 2019) or tide (e.g., Bowern, 2012). In such groups environment-based concepts are often favored for everyday spatial reference, even on small (so-called “table-top") scales (e.g., Pederson et al., 1998; Levinson \& Wilkins, 2006); reflected in gesture (e.g., Le Guen, 2011); mastered by children at young ages (De León, 1994); used in settings where they might seem inappropriate, such as indoors (Cooperrider, Slotta, \& Núñez, 2017); evoked in dreams (Evans, 2010); reflected in the orientation of houses and settlements (e.g., Núñez \& Cornejo, 2012); and folded into cultural practices like sleep and burial (Wassmann \& Dasen, 1998). Of special interest for present purposes, such contrasts are also used to construe elusive dimensions of human experience—dimensions like time.

Environment-based construals of time are of potential interest to a broad swath of fields-from linguistic anthropology to developmental psychology to cognitive neuroscience. And yet, despite this potential for broad interest, details about such systems remain sketchy and scattershot. The broad goal of the present paper is to remedy this. To this end, we present the fullest portrait yet of one environment-based system for conceptualizing time: the topographic system used in the Yupno valley of Papua New Guinea. In our initial report on this system (Núñez et al., 2012), our primary aim was to show that Yupno speakers really do conceive of time in terms of environmental rather than bodily contrasts - to show, in short, that such a system exists. We thus left a number of questions unresolved, including about the logic of the system, its linguistic realization, individual variation in its use, and its cultural entanglements and 
experiential motivations. In the present report, drawing on additional fieldwork-including further interviews and linguistic elicitation - we revisit these questions. We also make an effort to further contextualize this distinctive way of understanding time. The Yupno system-like all human conceptual systems - is best understood within the broader ecology in which its rooted, including the natural and built environment, linguistic resources and discourse regularities, and a suite of relevant cultural practices. We begin with a brief introduction to the Yupno valley and Yupno communities.

\section{The Yupno valley of Papua New Guinea}

The New Guinea region is a major world hotspot of linguistic diversity (Gorenflo et al., 2012), harboring an estimated 1300 living languages (Palmer, 2017). The Upper Yupno valley is part of the Finisterre Range; it straddles the border of Madang and Morobe provinces and is home to some 8000 speakers of Yupno, spread across approximately 25 villages (Slotta, 2015). Most people in the region are subsistence horticulturalists. Yupno (also spelled Yopno) is a Papuan language, grouped in the Nuclear Trans New Guinea family. Our joint research has been based in the village of Gua, which neighbors the administrative center of Teptep, and lies at an elevation between 2000 and 2200 meters above sea level (Figure 1).

The Yupno valley is formed by the Yupno river, which flows from its high-altitude source down to the Bismarck Sea. Off this main valley are a number of "side valleys" formed by smaller rivers that drain into the Yupno. Gua village sits in one such side valley, defined by the Daldal river (as well as the Jetjet river, which parallels it). These side valleys often have a common geophysical structure: a gentle slope defined by the flow of the smaller river into the 
Yupno, which we describe as the upvalley-downvalley axis; and steeper slopes on either side of the smaller river, from the nearby ridges down toward the side valley floor and river.

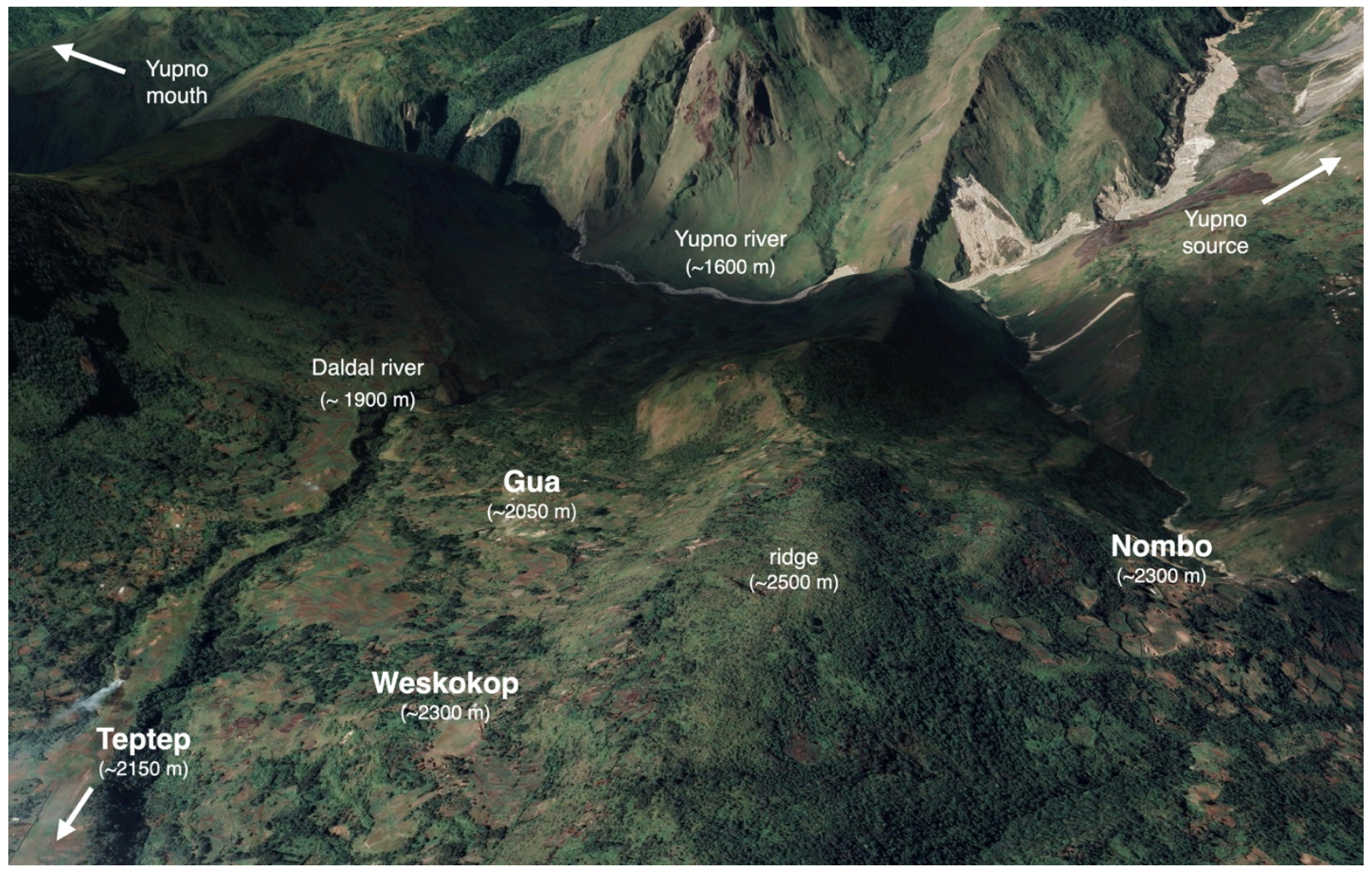

Figure 1. The region of the Upper Yupno valley where the present research was conducted. Interviews were carried out in three villages: Gua, Nombo, and Weskokop, each with a different topographic profile. In Gua, where much of our work has been based, there are two salient uphill-downhill axes: first, a relatively steep upslope-downslope axis, stretching from the Daldal river to the ridge above Gua; second, a relatively gentle upvalley-downvalley axis stretching from Teptep down to the Yupno river. From Gua, Nombo is a $60-90$ min walk $(\sim 1.7 \mathrm{~km}$ as the crow flies) and Weskokop is a 20-30 min walk $(\sim 1.3 \mathrm{~km})$. Elevations are shown in meters above sea level; the camera faces approximately southeast. Imagery: Google Earth.

The Yupno valley is rugged and remains relatively remote. No roads come into the area, so one must enter by foot—a difficult journey of a couple days from the nearest cities, Madang and Lae — or by small plane — a prohibitively expensive proposition for most. As a result of this relative isolation, flows of people and goods into and out of the valley are limited. There is no steady influx of print media, for instance, and while the world of contemporary global culture 
does seep in, it usually feels distant. Within the valley, there are no vehicles (animal-drawn, motorized, mechanical, or otherwise) or graded surfaces; people travel exclusively by foot to tend gardens, gather firewood, and visit neighbors over a network of footpaths (Figure 2). At the time of the present research, infrastructure was quite limited, with water available at communal taps but no electricity, phone service, or internet. Wood fires burn throughout the day and night, providing heat, light, and fuel for cooking.
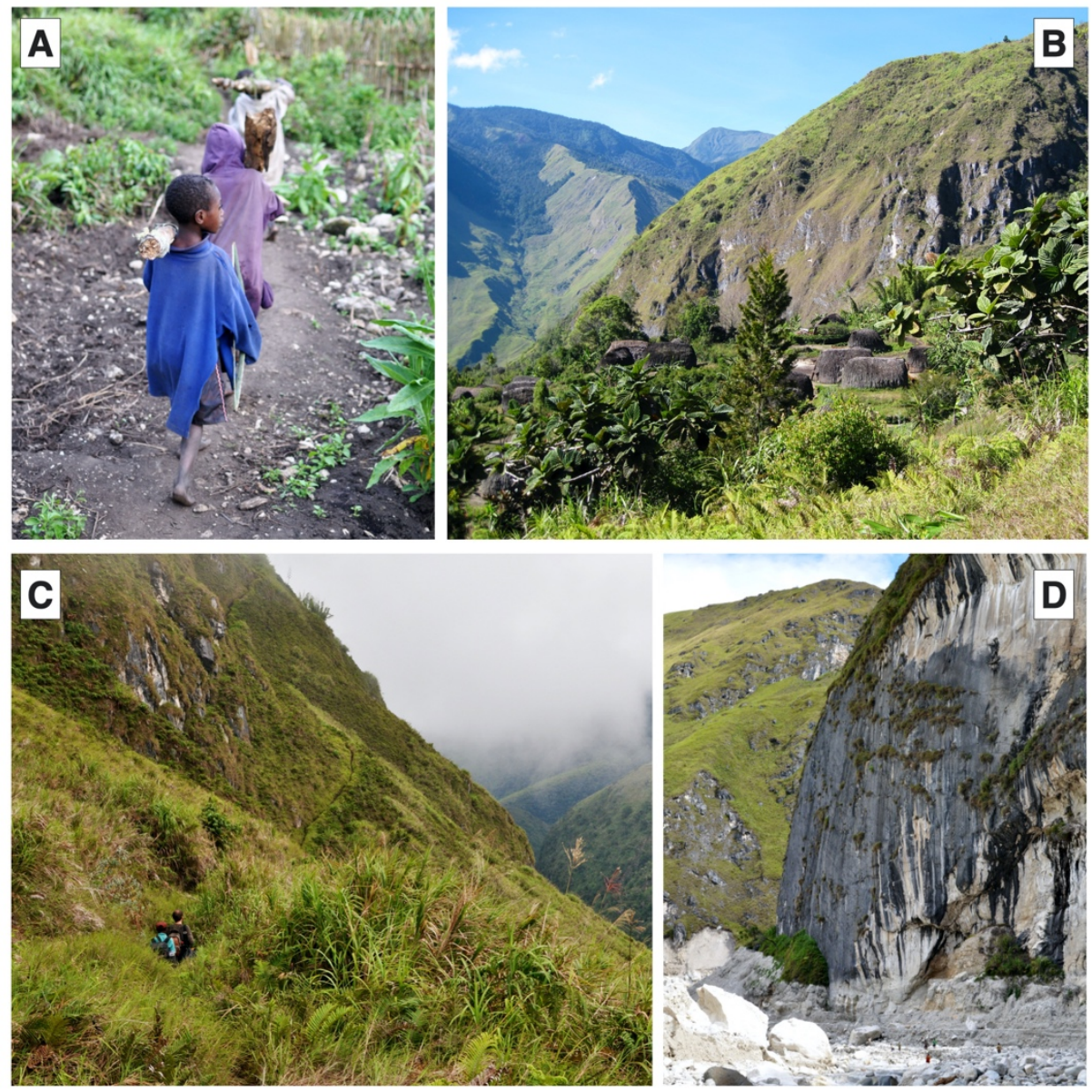

Figure 2. The lived topography of the Yupno valley. (A) A group of boys carry firewood back to the village. (B) A cluster of houses in Gua. The village includes dwellings spread over an elevation of roughly 200 meters (from 2000-2200 meters above sea level); walking to a nearby house thus often involves going up or down a slope. (C) Men on a path winding up and over a 
ridge toward the village of Nian. (D) The gorge of the Yupno river at its nearest point of access from Gua.

Despite this remoteness, the Yupno have undergone substantial cultural exchange with other parts of Papua New Guinea and the wider world. Missionization in the region began around 1930 and intensified in the 1950s (Wassmann, 1994); today most residents of the valley are at least nominally Christian. The first airfield in the region was opened in 1971 (Wassmann, 1994), and Tok Pisin — the English-based creole used throughout Papua New Guinea and one of the country's official languages - has been a fixture of schooling for roughly half a century. Today, most adults under the age of 60 years old have at least some fluency in Tok Pisin (Slotta, 2015), though Yupno remains the "mother tongue" learned from birth and the language most used in informal conversation. Many villages in the region, including Gua, have their own schoolhouses, which provide instruction through the middle grades. To pursue further education, students must leave the valley — at considerable expense — to attend a regional high school in a city. Some adults who did not attend high school nonetheless have a basic level of literacy, which is used primarily in church contexts.

A notable aspect of the valley is the architecture. Most buildings are single-room homes made from traditional building materials (Cooperrider, Slotta, \& Núñez, 2017). These structures - hereafter "traditional houses"-follow a basic template, with an oval floorplan, a door at one end leading up a few steps to a raised bamboo floor, and a long fireplace at the center running the length of the house; there are few if any windows. The houses can be quite largesometimes 15 meters from end to end (Wassmann, 1994) — and so can accommodate a sizable group. Everyday life thus unfolds principally in two settings: the rugged terrain of the out-ofdoors and the dark, firelit interiors of traditional houses. As we will see, both these settings- 
natural and built—figure prominently in the Yupno cognitive ecology of time and space. For further ethnographic details, see Wassmann (2016), Keck (2005), Slotta (2012, 2015), and Schmidt (1991).

\section{Topographic spatial concepts in Yupno language and culture}

The Yupno rely heavily on "topographic concepts" when talking about where houses and objects are located, how things are oriented, and where people are going (for examples, see Cooperrider, Slotta, \& Núñez, 2017; Slotta, 2014; Wassmann, 1993, 1994). Such concepts are used on scales large and small, even when the setting is veridically flat, and even when — to an outside observer-terrain seems beside the point. A similar reliance on topographic distinctions is reported elsewhere in New Guinea's interior (e.g., Fedden \& Boroditsky, 2012; Von Heeschen, 1982), as well as in the broader Pacific region (e.g., François, 2004; Senft, 1997) and across the world's mountainous regions (Urban, 2020), including in the Himalayas (e.g., Post, 2019), the Caucasus (e.g., Forker, 2019), and Mesoamerica (e.g., Soto, 2011). Our notion of "topographic" bears unpacking ${ }^{1}$. The relevant linguistic terms do not make explicit reference to slope or terrain, such as with a morpheme meaning 'hill' (as in English "uphill"); and, in fact, they are also used to refer to purely vertical relationships. More general terms like 'up' and 'down' may thus seem more appropriate, and we do gloss the terms this way in some contexts. However, elicitation with Yupno speakers suggests the terms are prototypically associated with terrain, with the tilts and contours of the "horizontal" plane ${ }^{2}$. They are linked, in other words, to

\footnotetext{
${ }^{1}$ Some authors label similar systems "topographic" (e.g., Post, 2019), while others favor "elevational" (e.g., Forker, 2019, 2020). Forker (2020) further distinguishes "general elevational" systems from "topographic elevational" systems.

${ }^{2}$ To provide an intuition about the claim here, consider English speakers' prototypical associations with "up" and "down." An English speaker may readily say that someone trudging up a hill is "going up"
} 
the plane of canonical human activity — a plane which, for Yupno speakers, is very often tilted (for similar claims, see Brown \& Levinson, 1993).

As noted, topographic systems are widely distributed around the globe, but, importantly, not all are the same. A critical division among such systems is seen in the difference between the Yupno system and the Tzeltal system, which is perhaps the best described system in the literature (Brown \& Levinson, 1993; Brown, 2012). In Yupno, topographic terms are used according to local slopes; in Tzeltal, by contrast, they may be used in this way but also often function as fully abstracted vectors like 'east' and 'west' (Brown \& Levinson, 1993). There is no similar "cardinal-like" use of 'uphill' and 'downhill' in Yupno, a fact with implications for how people talk and gesture about space and time.

The key claim about the Yupno — and about other groups that lean on topography_is that topographic concepts are not just present but privileged. Several strands of evidence support this idea. A first is the sheer quantity and variety of words that are inflected for topography, including many that belong to basic, "closed-class" parts of the lexicon. The Yupno demonstratives, for instance, are optionally but pervasively inflected for uphill-downhill relationships. There is a single proximal form (like English 'this'), but for the medial and distal demonstratives there are neutral ('that'), up ('that-up-there'), and down ('that-down-there') variants (Cooperrider, Slotta, \& Núñez, 2017, Table 1). The basic motion verbs 'come' and 'go' are similarly inflected (Cooperrider, Slotta, \& Núñez, Table 2); they also combine with other verbs (e.g., 'send,' 'walk,' 'put') such that a large portion of Yupno verbs ultimately become inflected for ups and downs. Finally, there is a rich class of spatial modifiers (similar to prepositions or adverbs in English) that convey different kinds of topographic relationships. These include, for instance, a

rather than "going uphill," but nonetheless the more canonical image associated with "going up" is of ascending vertically. 
contrasting pair of adverbs that refers to elongated objects oriented parallel to the slope of the hill (doydoy) or perpendicular to it (baratda), as well as monolexemic terms glossable as 'next-toon-above-side' (awijban) or 'next-to-on-below-side' (abijban) derived from the basic motion verbs.

A second strand of evidence lies in speakers' preferred ways of talking: topographic terms are not just abundant in Yupno, they are widely used over alternatives. Neutral forms are available — as noted — but speakers prefer to specify ups and downs when possible, and sometimes reject neutral formulations. We also find that speakers prefer to specify location and motion using topographic contrasts rather than other spatial contrasts (e.g., based on bodily axes or ad hoc landmarks), even on very small spatial scales (Cooperrider, Slotta, \& Núñez, 2017; Wassmann, 1994). In a recent study using a referential communication task - the 'Pig \& Corn,' a variant of the classic 'Man \& Tree' (e.g., Pederson et al., 1998)—Yupno speakers used topographic contrasts on $75 \%$ of trials when outdoors (accounting for $47 \%$ of all strategies used) (Cooperrider, Slotta, \& Núñez, 2017). Meanwhile spatial contrasts like 'left' and 'right'—which are heavily used by English speakers in similar tasks (Li \& Gleitman, 2002)—were vanishingly rare, despite the fact the arrays used were often laterally asymmetric (Cooperrider, Slotta, \& Núñez, 2017, p. 785).

A third strand of evidence is that topographic terms are frequently used beyond their semantic "home turf." For one, they are pervasively used on flat surfaces outdoors. This is done according to the slopes adjacent to the flat area; walking in the direction of a downhill slope, for instance, would be considered 'going downhill.' In the Pig \& Corn studies just mentioned, we showed that topographic contrasts are also widely used inside traditional houses (on $78 \%$ of trials and accounting for $35 \%$ of all strategies; Cooperrider, Slotta, \& Núñez, 2017). Such terms are 
used indoors by projecting a "conceptual topography" onto the house, with a longer uphilldownhill axis extending from the door (construed as 'downhill') to the opposite end of the house ('uphill') and two shorter uphill-downhill axes extending on either side of the house from the fireplace ('downhill') to the sidewall ('uphill'). This topography—which is reminiscent of the two-axis topography surrounding Gua (Figure 1) - is stable regardless of whether a house faces uphill or downhill relative to the surrounding terrain. It also shows up in how speakers gesture about time indoors: they generally locate the past toward the door and the future away from it (Núñez et al., 2012)3. To date we have only studied the phenomenon of "conceptual topography" in the case of houses, but Yupno speakers apparently also do something similar in urban environments such as the city of Madang, treating the major road — which is more or less flat—as though it defines an uphill-downhill axis (Wassmann, 1994, p. 660-1).

Still other strands of evidence support the privileged nature of topographic contrasts for the Yupno. They are mastered by children early in life—including being appropriately used indoors - and are incorporated into cultural practices such as conventional sleeping position (Cooperrider, Slotta, \& Núñez, 2017). Finally, they are also recruited for making sense of abstract dimensions of experience—notably, time, to which we now turn.

\section{Time in Yupno language and culture}

All languages have a more or less rich stock of linguistic resources for talking about different aspects of time and temporal experience. Here we consider these resources in Yupno,

\footnotetext{
${ }^{3}$ When we originally analyzed the time gestures in Núñez et al. (2012), we were unaware of the fact that the people also construe houses as having shorter uphill-downhill axes on either side of the fire (see Cooperrider, Slotta, Núñez, 2017). We suspect that speakers in fact construe time using both the longer and shorter uphill-downhill axes within the house, but confirming this would require new data or a reanalysis of the data in Núñez et al. (2012).
} 
with particular focus on those devoted to expressing concepts of duration, sequence time, and deictic time - the three major classes of temporal concepts that are construed spatially around the world (Núñez \& Cooperrider, 2013). Our work to date has zoomed in on the Yupno construal of deictic time - past, present, and future — so we will treat this class of concepts in the most depth, particularly when analyzing gestural examples.

The most generic temporal word in Yupno is bisap, a term meaning period or event, not unlike English "time." The word also means 'knot'; its reference to time may stem from earlier practices of tying knots to track events like new moons (Wassmann, 2016, p. 238). There are commonly used words for 'day,' 'week,' ‘year,' and 'season' (see Table 1). All are native Yupno terms except that for 'week' (sonda), which is Tok Pisin and rooted ultimately in English "Sunday." The Tok Pisin term for 'year,' krismas (from English "Christmas") is also commonly used. Both these Tok Pisin terms are metonymic in that a particular part of the week or year stands for the whole. The Yupno valley does not run on "clock time"; hours, minutes, and seconds are scarcely mentioned, if at all. Terms for days of the week and months are taken from Tok Pisin but are not commonly used; years are labeled according to the Western calendar but are not particularly culturally salient.

In comparing the magnitude of durations, Yupno speakers most often use words for 'long/tall' (jibagi) and 'short/small' (pisipmi). Long duration terms are frequently emphasized with an intensifier (-si); short duration terms are emphasized with the diminutive (-jok), which occurs as a morphological suffix and has several allomorphs (Cooperrider \& Núñez, 2012). The diminutive is also used to mark the precision of temporal intervals, a pattern that is common across languages (Jurafsky, 1996). 
Table 1. Key temporal lexicon in Yupno

\begin{tabular}{|c|c|c|c|}
\hline Word & Translation & Type & Notes \\
\hline bisap & time & unit & lit. 'knot' \\
\hline sigak & season & unit & \\
\hline bulak & year & unit & \\
\hline krismas & year & unit & Tok Pisin via English "Christmas" \\
\hline sonda & week & unit & Tok Pisin via English "Sunday" \\
\hline gildat & day & unit & \\
\hline apjok & today, now, present & deictic & \\
\hline apma & yesterday, last & deictic & \\
\hline usadon & tomorrow, next & deictic & lit. 'next dawn' \\
\hline jagare & two days ago & deictic & \\
\hline piday & two days from now & deictic & \\
\hline don & later, after & $\begin{array}{l}\text { deictic/ } \\
\text { sequence }\end{array}$ & \\
\hline kalip & previous, before & deictic & \\
\hline don-si-ngan & in the (distant) future & deictic & \\
\hline kalip-si-ngan & in the (distant) past & deictic & \\
\hline imi-gap-mi bulak & last year & deictic & $\begin{array}{l}\text { lit. 'down-side year'; may mean a } \\
\text { year or two }\end{array}$ \\
\hline isi-gap-mi bulak & next year & deictic & $\begin{array}{l}\text { lit. 'up-side year'; may mean a } \\
\text { year or two }\end{array}$ \\
\hline babik & ancestors & deictic & \\
\hline manjip & behind, after & sequence & $\begin{array}{l}\text { lit. 'back, rear' (as in a person's } \\
\text { 'back') possibly calqued from Tok } \\
\text { Pisin }\end{array}$ \\
\hline kwen & later, after & sequence & lit. 'above' \\
\hline
\end{tabular}

The topographic system enters into the expression of durations when describing the continuation of a process (what linguists refer to as the "durative"). Specifically, as a process unfolds it is said to 'go up' (there is no contrasting sense in which a process can 'go down') (see Slotta, 2014, p. 44-5, ex. 94-98).

Yupno speakers express sequential relations in several ways. Sequence time-sometimes known as "tenseless time" (McTaggart, 1908)—concerns earlier and later relations between events; it differs from deictic time in that it does not necessarily specify a now and thus does not concern past, present, or future. It is the type of concept at play, for instance, when describing 
different steps in a ceremony, seasons in a year, stages in the lifespan, or the acceleration of a falling object in physics, none of which are obligatorily anchored to now. Interestingly, Yupno furnishes linguistic resources for describing the 'after' relation but apparently not for describing the 'before' relation (note that many languages have terms for neither relation; e.g., Bohnemeyer, 2009). This apparent "before-less-ness" is evident in two conceptual models Yupno speakers commonly employ when describing sequences. A first is the one found worldwide and already discussed: the events-in-a-queue construal, based on a line-up of entities moving in the same direction. The entities correspond to temporal landmarks, such that later landmarks are said to be manjip (literally 'behind') earlier ones. However, Yupno speakers do not spontaneously use a contrasting spatial term for 'in front of' to mean 'before.' (It seems possible to extract such a usage during meta-linguistic discussion, but it does not occur in actual usage, nor is it readily volunteered.) In the other major conceptual model, later events are said to be kwen ('above') earlier ones (see Slotta, 2014, p. 29, ex. 44). Here, again, Yupno speakers do not spontaneously use the contrasting term mokgwan ('below') to mean 'earlier.' The imagistic origins of this second model may be related to the notion of a piling — with things added later on top of others - or perhaps growth (discussed later). One use of this second model shows up in how speakers link events together in discourse: one speaks of 'going up' from the first to the second event. Whether this conceptual model is the same one that motivates the idea that unfolding processes "go up" as they progress is unclear. Lastly, sequences in Yupno are commonly described using lists. These are often initiated with the Yupno term mibil-tok ('first') (mibil means 'base', 'root', or 'cause'), or with the Tok Pisin expression namba wan (rooted in English "number one"). 
In contrast to sequence time, deictic time involves construing events relative to now, and thus separating out past, present, and future. Yupno words that are inherently deictic include apjok ('today, now'), apma (yesterday), usadon (tomorrow, lit. 'next dawn'), as well as less frequent terms for 'day before yesterday' (jagare) and 'day after tomorrow' (piday) (see Table 1). Apma and usadon can also be appended to other temporal units to indicate 'last' or 'next,' as in apma sonda ('last week') or usadon krismas ('next year'). A frequently used word is don ('later, after'), often intensified as don-si. Overtly topographic words are somewhat rare in deictic time expressions, but they do occur. Particularly common is a pair of opposing deictic words: $i s \dot{t}$-gap-mí (dem.up-side-poss) and $\dot{i} m \dot{t}$-gap-mí (dem.down-side-poss), which combine with time units like bulak to yield, for instance, isi-gap-mi bulak ('a few years from now') and imi-gap-mi bulak ('a few years ago'). Also used are the demonstratives asudon (dem.up-loc) and amodon (dem.down-loc) to mean 'at that time (in the future)' and 'at that time (in the past).'

\section{Time in Yupno gesture}

The linguistic resources just described provide an initial sense of the spatial construals of time used in Yupno. But how speakers actually think about time on the fly shows up more vividly in their gestures. Gesture is universally produced in all cultures (Kendon, 2004; Kita, 2009); provides spatial information that goes beyond what is in speech (Alibali, 2005; Cienki \& Müller, 2008; Núñez \& Sweetser, 2006); and requires no special equipment to study (beyond a video camera). By examining spontaneous gestures, we can thus get a naturalistic view of how the topographic understanding of time stirs to life in the minds and hands of Yupno speakersand a more granular view than we could using other sources of evidence. 
The examples discussed here are taken from interviews conducted in July 2013. These interviews followed the semi-structured procedure described in Núñez et al. (2012): we first prepared a list of temporal expressions we thought would yield interesting temporal reasoning, with an emphasis on deictic time; we then recorded a native speaker of Yupno saying these phrases; and then finally played them during interviews and invited participants to explain them (in Yupno, though many also used some Tok Pisin). Gesture was not mentioned to participants ${ }^{4}$. Interviews were conducted in two segments, with the participant facing in different directions in each segment—e.g., first facing downhill then facing uphill—-to confirm they were gesturing according to the topography and not according to their own bodily axes. We conducted 10 interviews with a total of 14 adults ( $11 \mathrm{men})$. The preponderance of men is due to the fact that women are more likely to be engaged in garden work during the day, and also less inclined to talk to a group of male foreigners. In contrast to the interviews reported in Núñez et al. (2012), which were all recorded in the central market in Gua, our 2013 interviews were filmed at three villages: Gua (three interviews); Nombo (four interviews), and Weskokop (three interviews) (see Figure 1). These additional villages were selected to help us better understand the nature of the uphill-downhill concepts being used, specifically whether speakers were construing time according to local slopes or according to the macrolevel inclination of the valley, which follows the downward flow of the Yupno river to the sea. In two cases, we conducted interviews with the same speaker in two different villages to better understand how their construals of time might change, or not, across settings.

\footnotetext{
${ }^{4}$ Because this procedure generates focused discussion of time concepts, it likely elicits more temporal gestures than would be observed in informal conversation. However, research in other groups (e.g., Valenzuela et al., 2020) has shown that temporal gestures also occur in naturalistic conversations and we have no reason to doubt they do in Yupno as well.
} 


\section{Example 1. WI in Gua}

A first sequence of interest is taken from an interview in Gua. A group of men - two in frame and several others off-camera — sit facing upvalley, toward the neighboring village of Teptep (Figure 1). The man at right, WI, has been asked to explain the term don, meaning 'later' or 'future.' The sequence begins with WI drawing a distinction between 'now' —-which he locates with a quick downthrust of his right hand in front of him —and 'later' — which he locates upvalley, ahead of him and high above. The angle of his upward gesture is so steep, in fact, that it leaves the camera frame; it is notably exaggerated relative to the actual topography, which inclines gently into the distance (Figure 3). In all transcribed examples, temporal gestures are marked by underlining, and those gestures depicted in the associated figure are numbered with superscripts; these numbers correspond to the figure panel in which they appear.
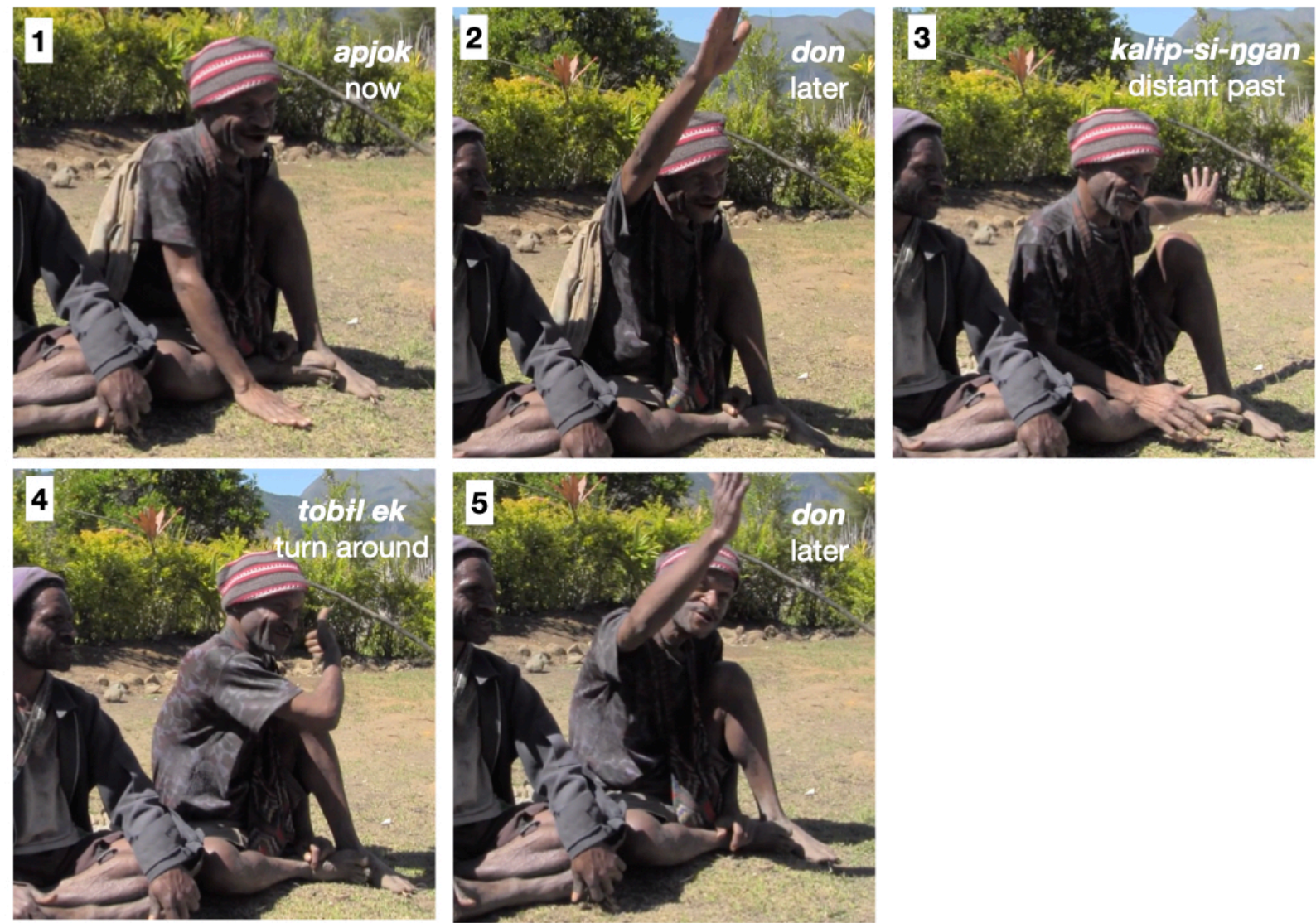
Figure 3. Five temporal gestures from Example 1. The speaker locates the present where he is sitting (panel 1), the future upvalley and above (panels 2, 5), and the distant past downvalley (panel 3, 4). In all figures, panel numbers correspond to the words numbered with superscript in the transcript; the panel shows the speaker's gesture while producing the numbered word.

WI: don yan on

later thus dem

"'Later," it's like this'

apjok ek-amay-da don ko-kda-may

now be-1p.pres-subj later go-imm-1p.pres

'Now, here we are and then we are about to go to later'

WI then repeats the contrast between 'now' and 'later,' also repeating the associated gestures. His 'now' gesture takes on a different character in its second version: rather than a loosely formed "chop," he thumps his full hand, palm open, down onto the ground (Figure 3.1); his 'later' gesture is nearly identical to the first (Figure 3.2).

WI: apjok $^{1}$ ini yik-gaman-da e now here sit-1p.pres-subj and

'Now we are here and then'

$\underline{\text { don }}^{2}$ ko-kda-may

later go-imm-1p.pres

'We will go to later'

WI then elaborates his spatial model of time by adding in the past. He does this with his

left hand, placing the ancestors downvalley, behind him and to the left, toward the gorge of the

Yupno river (Figure 3.3).

WI: akan babik kalip-si-ngan ${ }^{3}$ yogop-git

and ancestors before-emph-into be-3p.past

'The ancestors were in the past'

JS: kalip-si-ngan ko-kda-man

before-emph-into go-imm-1p.pres

'We will go into the past?' 
One of us, JS, asks about the phrasing of 'we will go into the past,' implicitly questioning whether this would be a reasonable thing to say. WI repeats the phrasing, twisting around slightly and thrusting his right hand repeatedly over his left shoulder (Figure 3.4), in the direction of the recently located ancestors.

\section{WI: kalip-si-ngan ko-kda-man before-emph-into go-imm-1p.pres}

"We will go into the past""

on awi-nin tobil $\mathrm{ek}^{4}$ babik-gan ki-ney

dem again-1p.poss turn do.SS.CC ancestor-into go-1p.irr

'That means we will turn around and go again into the time of the ancestors'

After a brief bout of commentary from the others, WI declares the suggestion impossible. The sequence ends with him reiterating that 'later is where we will go'; his gesture and gaze together emphasize the steep uphill trajectory of this future-ward journey (Figure 3.5).

\section{WI: jiden ek ki-ney on aripmi dima how do.SS.CC go-1p.irr dem possible neg 'How will we go, it's impossible'}

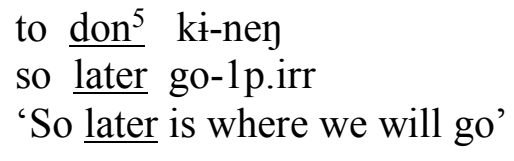

The sequence illustrates several key features of topographic construals of time in Yupno. It shows the assignment of the three deictic time categories to different spatial positions - the present where the man is sitting, the past downvalley, and future upvalley and above. It also shows that Yupno time-related gestures often contain spatial information that goes beyond what is contained in speech. Aside from the general idea that the future is somewhere we are 'going' and the past somewhere we could 'turn' toward, there is nothing in speech that explicitly locates 
the future or past with respect to the terrain. This happens entirely in WI's gestures. Indeed, even if he had been using the topographically inflected forms of the verb 'to go' when describing 'going' into the future or past, his gestures would still lend specificity to the idea: 'going up,' again, can mean anything from a specific direction along the horizontal to purely vertical, but WI's gestures show he is construing the future as somewhere in between. Finally, the example hints at an interesting degree of variability in Yupno time construals. Here, WI is imagining the future as upvalley, along the gentle slope up toward Teptep, and the past as downvalley, along the gentle slope down toward the Yupno river. This construal differs subtly from the prevailing construal suggested by the data in Núñez et al. (2012), in which the past is associated with the mouth of the Yupno river and the future with its source (p. 30, Figure 2B). Both patterns are broadly consistent with a future-uphill construal, but WI's gestures provide a first hint, confirmed throughout our 2013 interviews, that Yupno speakers vary in which sense of uphill and downhill they adopt.

\section{Example 2. KA in Gua}

The next example involves the same group of men as Example 1 but facing the opposite direction, downvalley toward the gorge of the Yupno river. The speaker is the man on right, KA. He is explaining a sequence of three terms: jagare ('day before yesterday'), apma ('yesterday'), and apjok ('today, now'). The first time he runs through the sequence, he produces three gestures at different positions in front of his body: furthest away with 'day before yesterday,' a bit closer in with 'yesterday,' and finally almost in his lap with 'now' (Figure 4.1-3). Again, the locations of temporal gestures in the transcript are marked by underlining. 

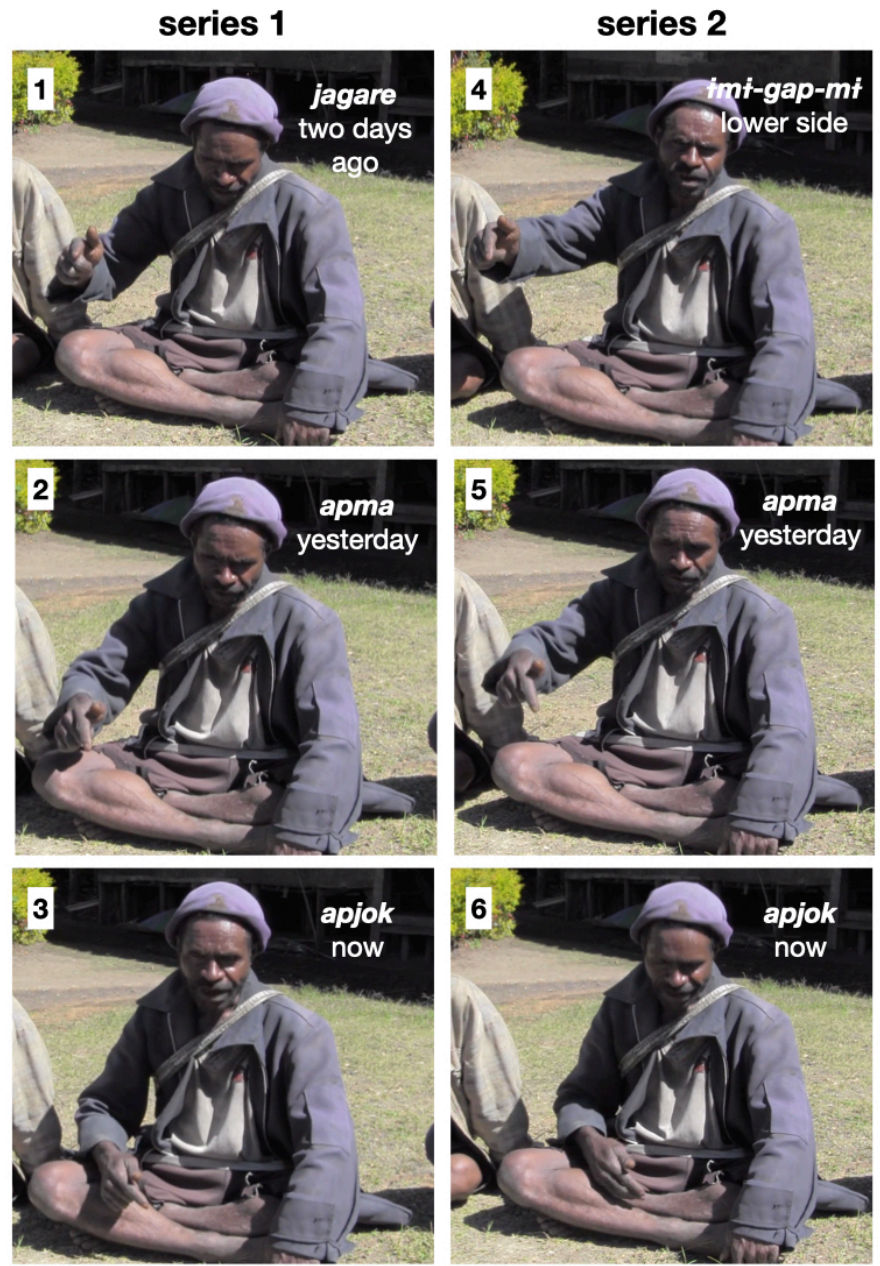

Figure 4. Six temporal gestures from Example 2. The speaker, facing downvalley, twice produces a series of gestures (panel 1-3; panel 4-6) to accompany a three-term sequence: 'two days ago' (panel 1, 4), 'yesterday' (panel 2, 5), and 'today' (panel 3,6).

KA: kili jagare $^{1}$ yogop-guman-da e

disc day.before.yesterday be(anim)-1p.past then

'We were at the day before yesterday and then'

apma $^{2} \quad$ yogop-guman-da e

yesterday be(anim)-1p.past then

'We were at yesterday and then'

apjok $^{3} \quad$ e-kaman

and now be(anim)-1p.pres

'We are at now' 
All three are produced with his right hand in a loosely formed index-finger-extended handshape. He repeats the sequence, but this time uses overt spatial language, imi-gap-mi ('down side'), to replace the term jagare (Figure 4.4-6):

KA: kili inun imi-gap-mi-kon ${ }^{4} \quad$ yogop-guman-da disc dem dem.down-side-poss-loc be(anim)-1p.past-abs

'Alright, we were on the lower side (day before yesterday) and then'

apma $^{5} \quad$ yogop-guman-da yesterday be(anim)-1p.past-abs

'We were at yesterday, and then'

apjok $^{6}$ ini e-kaman

now here be(anim)-1p.pres

'Here we are at now'

Like Example 1, this sequence shows a speaker gesturally assigning three different temporal landmarks to three different spatial positions. Here, however, the construal is of three different degrees of deictic remoteness — construed as different distances from the speaker's sitting position—rather than three different deictic time categories. As in Example 1, KA's construal unfolds along the upvalley-downvalley axis. This provides further evidence that Yupno speakers do not uniformly construe time with the past toward the mouth of the Yupno river and the future towards its source. Rather, the future-uphill construal appears to be more flexible, with speakers keying on whichever slopes are most salient in the speech situation; this is likely determined by the particular landform one is sitting on and how one is oriented, among other factors. Finally, Example 2 also shows how qualitatively similar gestures may be accompanied by overt spatial language (Figure 4.4) or not (Figure 4.1). 


\section{Example 3. WI in Nombo}

The next example features the same speaker as Example 1, WI, but in a different village: Nombo. Nombo is located $60-90 \mathrm{~min}$ by foot from Gua; getting there requires ascending a prominent ridge - roughly 450 meters higher than Gua — and then descending on the other side (see Figure 1). We traveled to Nombo because its geophysical setting would shed further light on whether Yupno speakers key on the macrolevel inclination of the valley or rather on more local slopes when construing time. In Nombo, if one were to place the past in the direction of the mouth of the Yupno river, one would thus be gesturing uphill with respect to a prominent local slope; if one were to place the future in the direction of the river's source, one would be gesturing toward a steep, nearby downhill.

In this sequence, WI is contrasting some of the same concepts as in Example 1. He begins by explaining the term kalip-si-ygan ('distant past'):

\begin{tabular}{|c|c|}
\hline \multirow[t]{4}{*}{ WI: } & 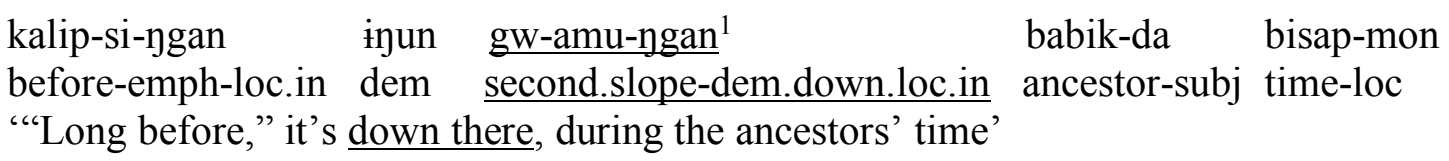 \\
\hline & $\begin{array}{l}\text { bo } \begin{array}{l}\text { ik-amu-ngan } \\
\text { or }\end{array} \text { babik-si-ngan } \\
\text { or } \underline{\text { straight-dem.down-loc.in ancestor-emph-loc.in }} \\
\text { 'Or straight down, during the ancestors' time' }\end{array}$ \\
\hline & $\begin{array}{l}\text { akan } \underline{\text { don-si }}^{3} \text { inun gw-asu-ngan } \\
\text { and } \underline{\text { later-emph dem second.slope-dem.up-loc.in }} \\
\text { "And "much later," it's up there' }\end{array}$ \\
\hline & 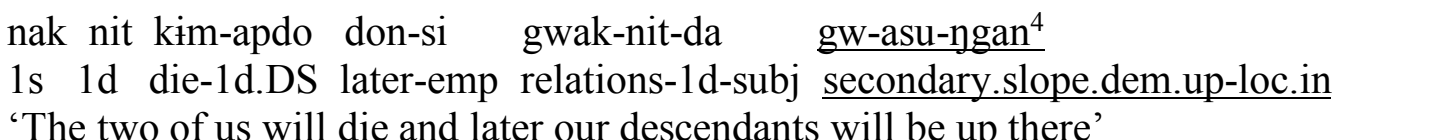 \\
\hline
\end{tabular}


He gestures twice to locate the distant past and ancestors far downvalley (Figure 5.1-2), toward the gorge of the Yupno river and then twice to locate the distant future far upvalley, away from the gorge (Figure 5.3-4).
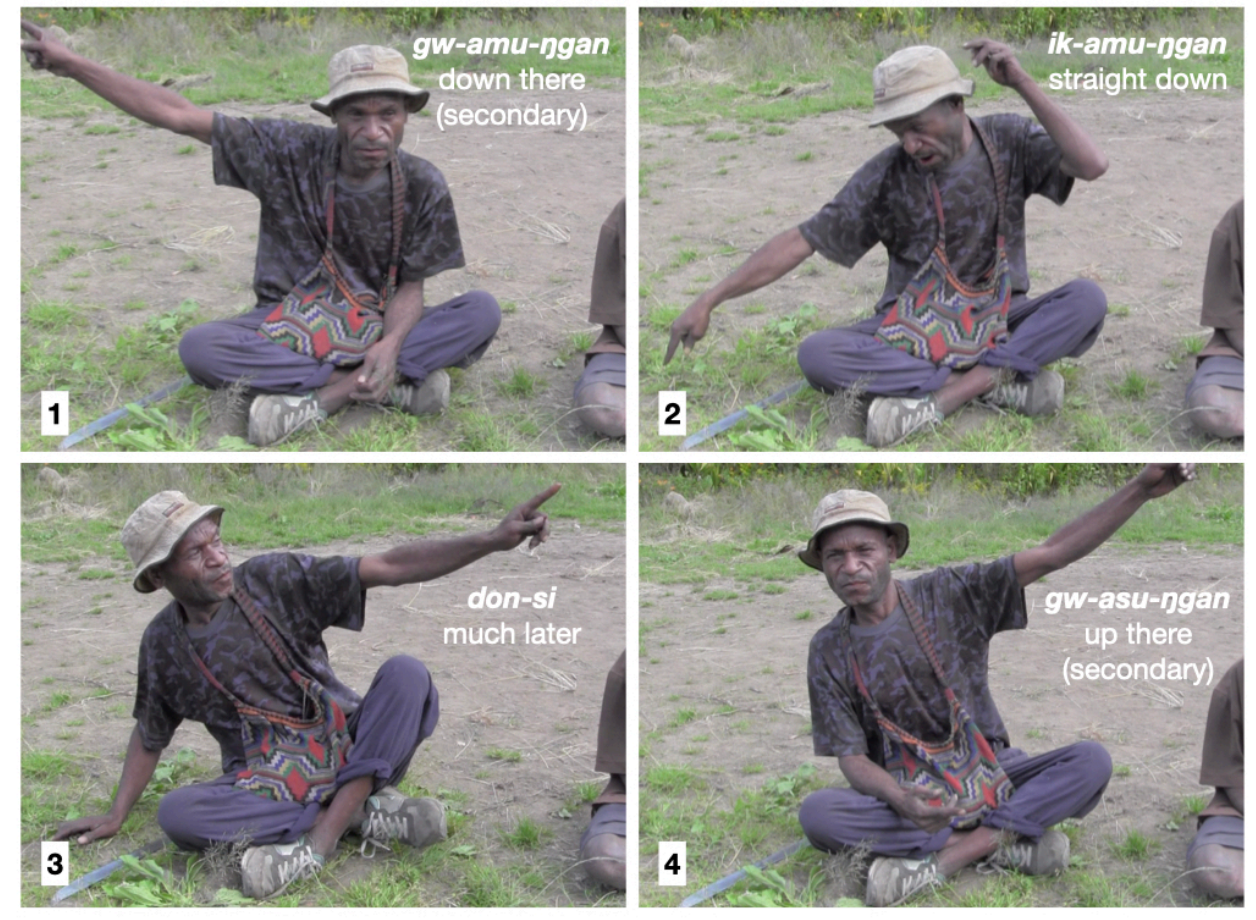

Figure 5. Four temporal gestures from Example 3. The speaker uses explicit spatial language, locating the distant past downvalley (panel 1,2) and the future upvalley in the distance (panel 3 , 4).

WI's gestures are, as in Example 1, markedly effortful—for instance, involving full extension of his arm - and, in two cases, involve looking toward where he is gesturing (Figure 5.2 and 5.3). This time his use of topographic terms is explicit. Each of his gestures is accompanied by a topographically marked demonstrative: in three cases he uses the lowfrequency topographic demonstratives that refer to the secondary (or gentler) slope, most often the upvalley-downvalley axis. These involve the addition of $g w$ - to the more basic demonstrative set (Cooperrider, Slotta, \& Núñez, 2017, p. 796). In Nombo, as he did in Gua, he’s gesturing 
along an axis extending toward and away from the gorge of the Yupno river - rather than toward the mouth and source of the river.

\section{Example 4. AR and ER in Weskokop}

The next example also involves gestures co-produced with overt spatial language. The sequence comes from an interview with two men, AR and ER, in Weskokop, a village that neighbors Gua to the northwest. The village is $20-30 \mathrm{~min}$ by foot from Gua and lies approximately 250 meters higher in elevation. We traveled to Weskokop to get, again, a richer sense of how the construal of time changes around the valley. Of interest in this sequence is the fact that, in contrast to the previous examples, the key gestures are produced with the head and face. Non-manual gestures are common in Yupno communication (Cooperrider et al., 2018), but were excluded from our prior work on time because they convey less precise spatial information (Núñez et al., 2012). The current sequence is repetitive, with both men making several head gestures upslope (toward their left) when using the phrase isi-gap-mi bulak ('future year') and downslope (toward their right) when using the contrasting term, imi-gap-mi bulak ('past year') (Figure 6). These phrases are similar to the English phrases 'next year' and 'last year' but do not make specific reference to the immediately previous or coming year. The future gestures of ER are of particular interest: they involve a stylized "looking up" in the direction of the slope rather than a mere toss of the head (Figure 6.2). Interestingly, here the axis at play is not the upvalleydownvalley axis, as used in the previous three examples, but the steeper upslope-downslope axis. The sequence thus provides further evidence that the choice of slope to anchor one's time references is determined locally and is not uniform across settings or speakers. 

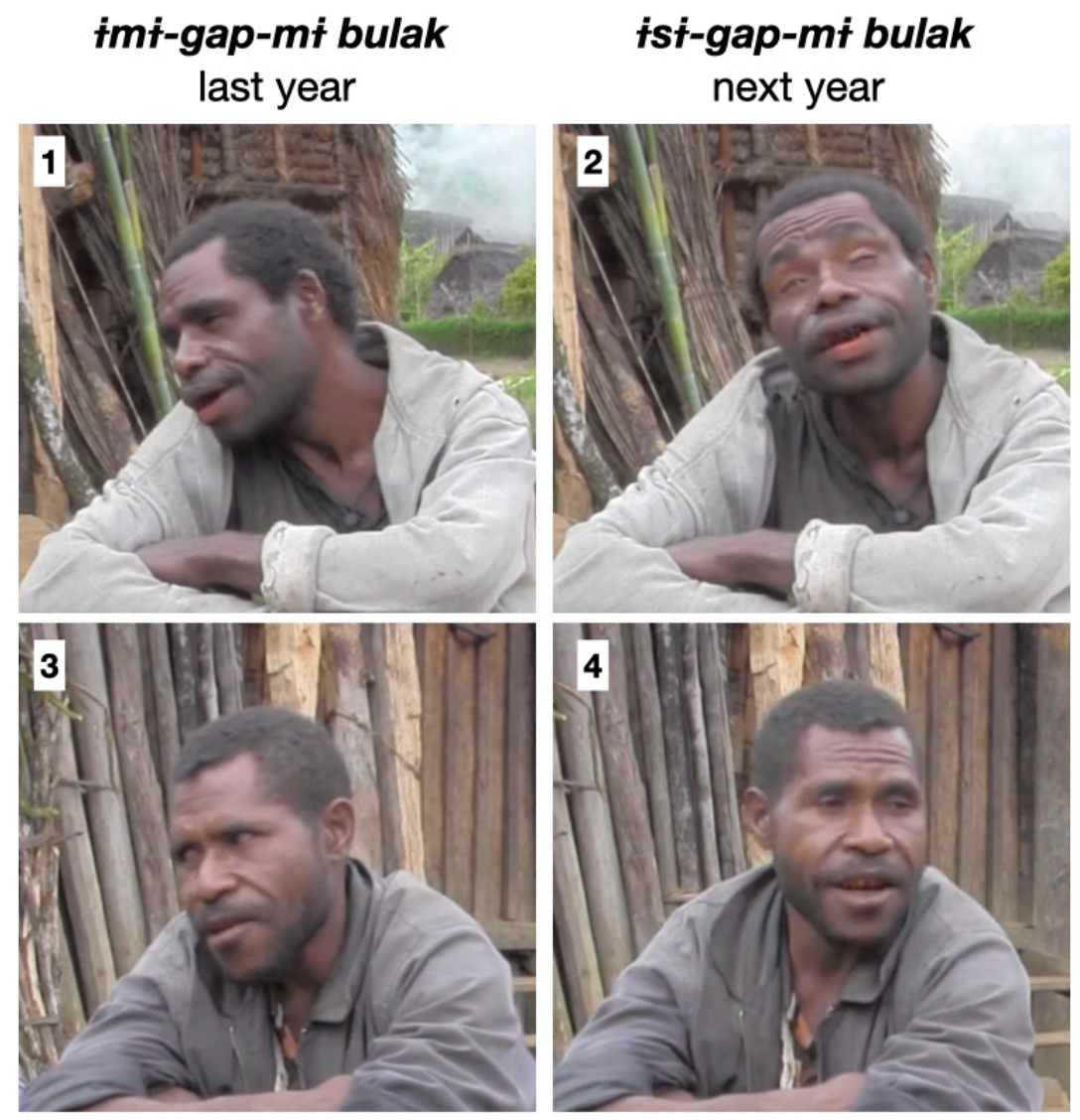

Figure 6. Four temporal gestures from Example 4. Both men repeatedly anchored references to the past with downslope head gestures (panel 1,3) and to the future with upslope head gestures (panel 2,4). The upslope gestures of the man in panel 2 involve a stylized "looking up."

\section{Example 5. KO in Weskokop}

The next example also comes from Weskokop. The man on the right, $\mathrm{KO}$, is contrasting terms spanning all three deictic categories. He arranges them in three contrasting locations, locating 'last year' downslope (behind him), 'this year' at his feet, and 'next year' to upslope and above (to his right) (Figure 7, sequence 1). The speaker interleaves his explanation with Tok Pisin, marked below in italics:

KO: imi-gap-mi bulak on las yia ${ }^{1}$ dem.down-side-poss year dem last year 'The year below is last year' 


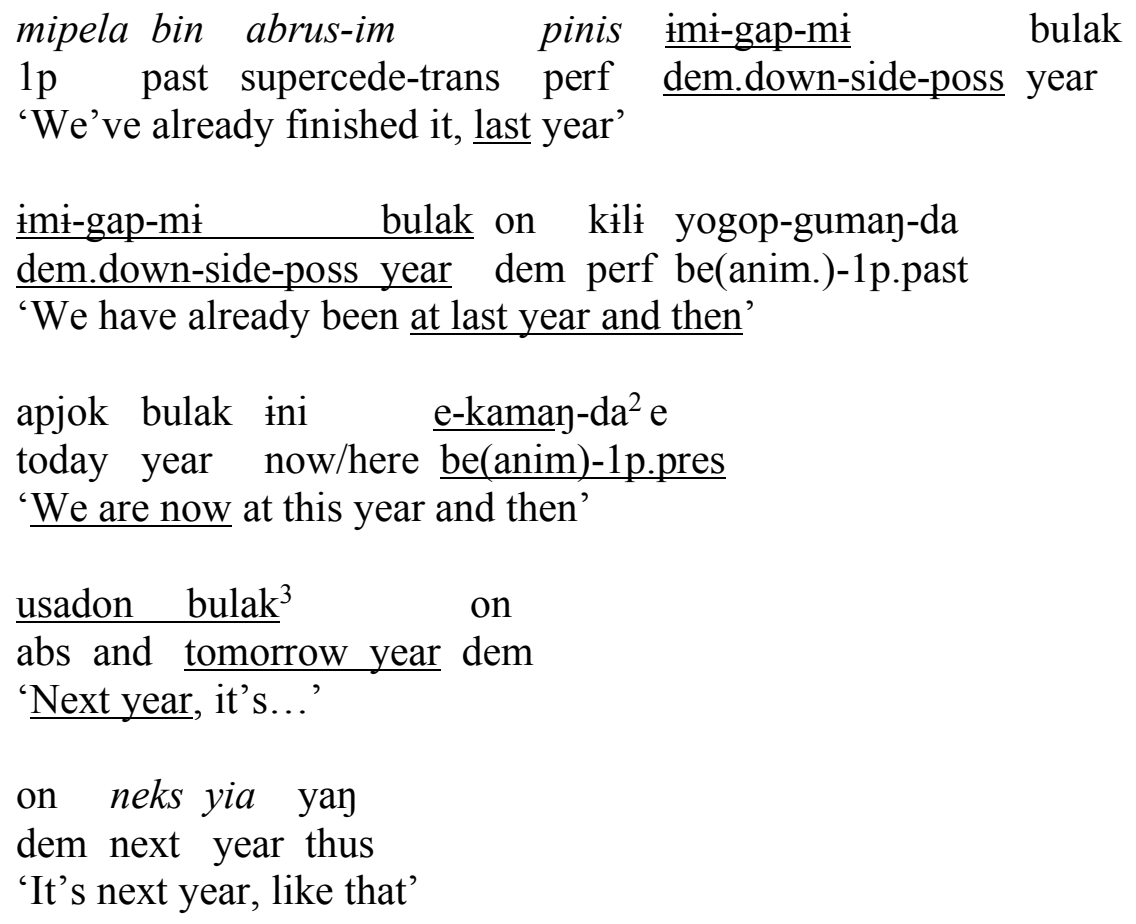

The example shows the systematic use of space to contrast landmarks in the three different time categories. KO seems to be keying on the same upslope-downslope axis as the men in Example 4. The example also shows again the same interesting asymmetry, seen in Examples 1 and 4, with the future located steeply above and the past located roughly along the horizontal. 

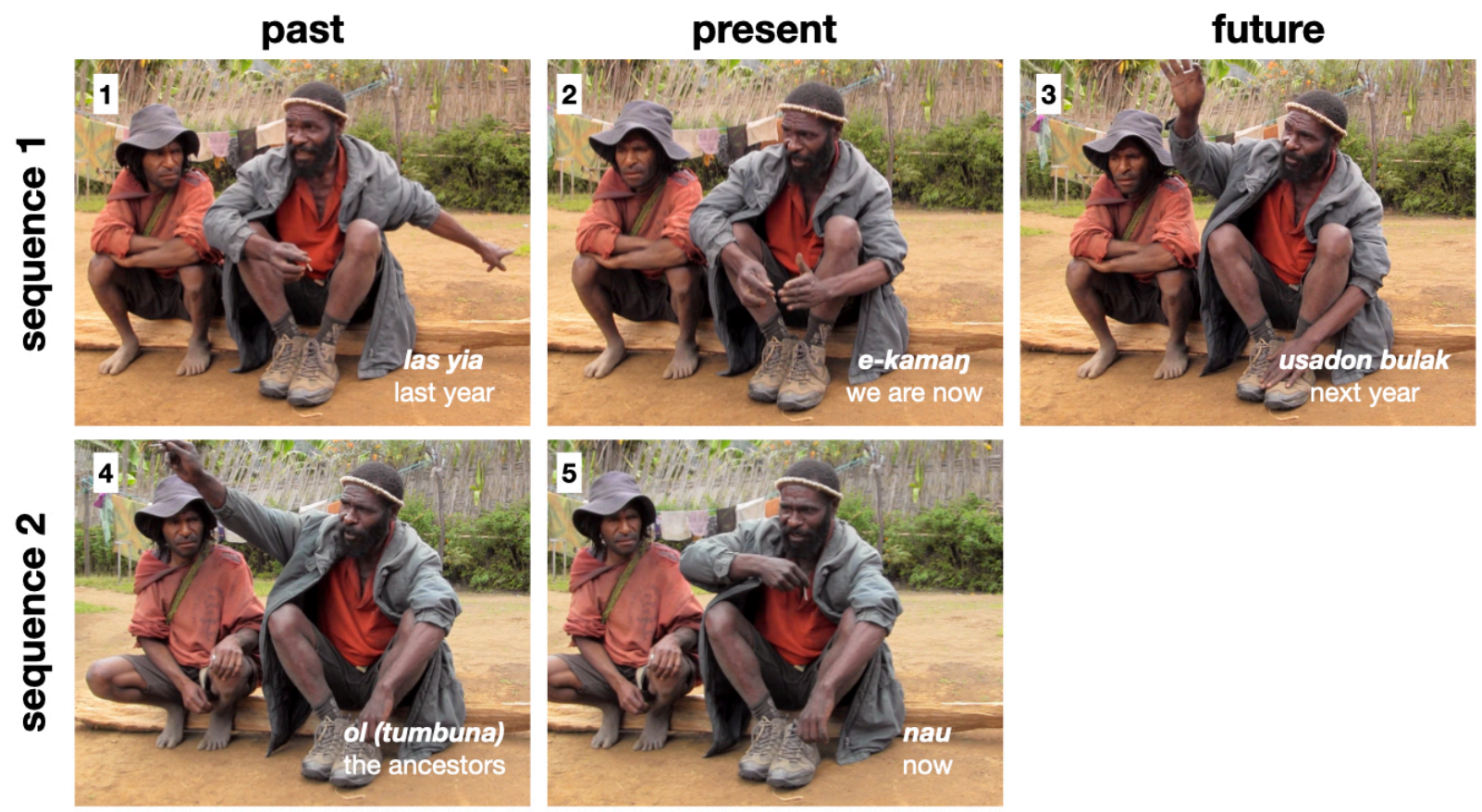

Figure 7. A speaker produces five temporal gestures across two separate sequences. In both sequences he locates 'now' where he is sitting. However, whereas in the first sequence he locates the past downslope (panel 1) and the future upslope (panel 3), in the second he locates the past upslope (panel 4). The speaker thus did not maintain a consistent future-uphill construal of time.

\section{Example 6. KO in Uskokop}

The systematic use of space in Example 5, however, proved to be the exception for KO. More often he gestured according to a reduced model of time in which past and future contrast with now, but not with each other. This might be considered a future-is-elsewhere construal of time. Interestingly, such a generic mapping is found in the semantics of deictic terms in the world's languages (e.g., in Urdu; Tillman et al., 2017) and has previously been attested in temporal gestures (Le Guen \& Balam, 2012). The non-contrastive nature of KO's past and future gestures is evident in a second sequence in the same interview, beginning about three and a half minutes later (Figure 7, sequence 2). Here again he interleaves Yupno and Tok Pisin (in italics). Since the interview is the same, numbering continues from Example 5: 
KO: babik on kalip amin

ancestors dem before people

'The ancestors were the people who lived before'

ol tumbuna tumbuna tru taunbilo $i$ stap taunbilo ya

pl ancestor ancestor emph down-below be down below disc

'The distant ancestors are down below'

on kalip-gwan babik

dem before-loc.in ancestor

'Those are the ancestors from before'

mipela no save nem bilong ol

$1 \mathrm{p} \quad$ neg know name poss $3 \mathrm{p}$

'We don't know their names'

from $\mathrm{ol}^{4}$ nau mipela kam kam kam kam and nau ${ }^{5}$ mipela stap

from $3 \mathrm{p}$ now $1 \mathrm{p} \quad$ come............. and now $1 \mathrm{p}$ be

'From them now we have come come come come and now here we are'

When locating the 'ancestors,' KO places them far in front of him and to his right — that is, in the same location upslope where, in the Example 5, he had placed 'next year.' This is all the more remarkable for the fact that he is using the Tok Pisin term for 'down below' while producing this gesture. Such an inconsistency might be easier to dismiss were the gesture a one-off or casually produced; but, in fact, the gesture is repeated several times and is clearly articulated. At one point, he represents the trajectory form the ancestors (Figure 7.4) to 'now' (Figure 7.5), tracing an arc with his right hand from its full extension to his right and above into his lap. His gaze follows his hand as it moves, underscoring the gesture's communicative relevance (e.g., Cooperrider, 2017). In a later interview in Gua, KO showed a similar inconsistency in gestures for past and future, but always preserved the basic mapping between distance from himself and "distance" in time.

KO is not the only Yupno speaker whose construal of time differs from the prevailing topographic model. As mentioned in our earlier report (Núñez et al., 2012, p. 29), we previously 
interviewed a man who consistently gestured according to a body-based model of time. This was evident across several interviews conducted at different locations in Gua, indoors and out: in all cases, he located the future behind him and the past in front of him, the past-in-front construal found in Aymara. These "non-conforming" construals of time may be due to any number of factors, such as where one was raised in the valley, where one has traveled, and which other spatializations of time one has been exposed to. But, generally, there may also be good reasonsdiscussed below - why idiosyncratic temporal construals of this sort may be more common in the Yupno context than they appear to be among, for instance, Euro-American undergraduates.

A few key generalizations emerge from these examples. First and most basically, they illustrate that the Yupno topographic construal of time-in which present is collocated with the speaker, the past is downhill from the speaker, and the future is uphill - is commonly expressed in gestures. Such gestures are not merely oriented along the horizontal in the direction of nearby slopes. Rather, future gestures are often pitched sharply upwards, and thus seem to embody slopes in their very form. (Interestingly, past gestures do not often embody an opposite downward slope; but see Figure 5.2). Second, the examples illustrate that time gestures are sometimes joined with overtly topographic language but often not; this suggests the system is cognitively vital and not merely yoked to certain linguistic expressions. Third, the examples support a key update to our understanding of the Yupno construal of time: the particular uphilldownhill involved is not necessarily based on the source-to-sea axis of the Yupno river. Rather, it appears to be more locally anchored and situationally selected. Depending on where one is, the most salient uphill and downhill for the purposes of time construal may be upvalley-downvalley or upslope-downslope. Fourth and finally, there is an intriguing degree of individual variability in how Yupno speakers construe time. This is seen, first, in the fact that speakers have latitude to 
construe past and future according to different senses of uphill and downhill. It also seen in the fact that some speakers — such as KO in Example 5 and 6 and the "outlier" participant described in Núñez et al. (2012) — depart from the future-uphill mapping altogether, while still showing a basic impulse to spatialize time.

\section{Discussion}

Our main aim here has been to fill out the picture of Yupno time concepts and, in the process, offer the most complete portrait to date of an environment-based way of thinking about time. In this section, we step back from the goal of describing the Yupno system and move to a trickier task: explaining it. We first consider how the system is rooted in — and sustained by —a particular cognitive ecology; we then consider why the system has the structure that it does, with the past construed as downhill and future as uphill.

\subsection{The cognitive ecology of the Yupno valley}

The Yupno time system can only be understood in terms of the broader cognitive ecology in which it is embedded - that is, the suite of environmental, cultural, and social elements that shape and scaffold how Yupno people think, act, and communicate (e.g., Hutchins, 2010; Marghetis et al., 2020; Núñez \& Cornejo, 2012). Much research over the last century, following Whorf (1956), has privileged language as a "prime mover" in shaping concepts of space and time across cultures. But more recent work has begun to tease out a broader set of relevant factorsfrom the geophysical environment (e.g., Palmer et al., 2017) and subsistence strategies (e.g., Shapero, 2016), to graphical practices (e.g., Danziger, 2011) and architecture (e.g., Núñez \& Cornejo, 2012). Here we discuss those elements of the cognitive ecology of the Yupno valley 
that seem especially important in nurturing and reinforcing the topographic construals of time we have described.

A first element is, of course, the topography itself. Slopes are visually and experientially salient in everyday life. Yupno people spend a good portion of any day in transit - to gardens, schools, friends' homes — which means they spend a good portion of any day walking up or down footpaths, some quite steep (Figure 2). Another key element—building on the first—is the centrality of topography in the Yupno language. Topography is enshrined in grammar, baked into the contrastive options a speaker must navigate when using a demonstrative or verb, for example. It is also part of discourse norms that quietly urge the use of topographic contrasts over other available options. Put together, these grammatical facts and discourse norms probably account for why Yupno speakers continue to use the topographic system when indoors. But the "conceptual topography" of the house is itself contingent on another aspect of the Yupno ecology: architectural uniformity. More variable housing styles would make such a shared system impractical. Together these three elements - salient topography, linguistic centrality, and architectural uniformity — reinforce each other to keep the topographic system activated in the Yupno mind throughout daily life. A fourth element may also contribute to the vitality of the topographic system: gestural practices, which can reinforce and perpetuate cultural models (e.g., Le Guen, 2011). As we have seen, Yupno speakers create rich and vivid representations of ups and downs with their hands, sometimes doing so when there is no hint of topography in their speech. Thus topographic notions - even when they are not verbalized — are present and sometimes foregrounded in Yupno communication.

In most other groups where time construals have been studied, graphical practices form a key part of the cognitive ecology. Wherever they are found, calendars, timelines, and (implicitly) 
text itself play a remarkably powerful role in "regimenting" spatial construals of time and other abstract concepts (Cooperrider, Marghetis, \& Núñez, 2017). These practices entail a canonical position by which the observer views the representation; in this way they encourage body-based ways of thinking about time as proceeding from the observer's left to right, right to left, or top to bottom, depending on the script or graphical convention. Yet such practices are a relatively limited, uneven presence in the Yupno valley. Western calendars are occasionally found—and are used to track church events, for example—-but are recently introduced and hardly ubiquitous. Nor are there older Yupno practices for representing time as space — at least none that play a prominent role in everyday life at the time of this research. (As mentioned, there was once a practice of tying knots to track events; and, in the village of Nokopo, there was a practice of projecting key times of year onto a salient mountain ridge [Schmidt, 1991, p. 42-44]. We have never encountered either practice in our research.) The scarcity of such practices in the Yupno valley may be critical to allowing a non-body-based way of thinking about time to flourish. This scarcity may also go some way in explaining the degree of individual variability we have observed—variability which, impressionistically, seems greater than we have encountered in studies with American undergraduates (Cooperrider \& Núñez, 2009; Walker \& Cooperrider, 2016). Many of the Yupno adults we interviewed have some exposure to schooling, and some degree of literacy, but they are hardly immersed in a world of printed and digital text. They are thus only exposed to the topographic construal of time through linguistic expressions - which are relatively infrequent, not to mention often ambiguous — and each other's gestures — which are fleeting and sometimes inconsistent. As a result, Yupno speakers have relative freedom to construct their own versions of the future-uphill mapping-or, indeed, to construct other spatial mappings entirely. 
Viewing the Yupno topographic system in an ecological light, as involving a set of mutually reinforcing elements, also underscores its fragility. A change in any of the elements just discussed could have cascading effects, potentially undermining the stability of the entire conceptual system. If more of the Yupno valley were flat and graded, for example, topography would become less experientially salient. If new building styles were introduced, conventions for talking about indoor space topographically may be simplified or abandoned. If more Yupno people were to learn to read and write through exposure to formal schooling, body-based ways of thinking about time would likely enter the ecology and compete with environment-based ones. If more people were leave the Yupno valley to settle in urban areas-with radically different terrain and building styles - it's not clear how much of the topographic system, if any, would survive the move (see, e.g., Schlossberg, 2018). None of these are negative developments on their own, of course. Nor are they idle hypotheticals; several of these changes are underway.

\subsection{Why is the future uphill?}

Even as we have filled in the picture of the Yupno topographic system, certain key details remain fuzzy or puzzling. Probably the most vexing open question, the one we get asked more than any other, is: Why is the future uphill? Why wouldn't tomorrow be 'down below' rather than 'up above'? In the case of every other temporal construal described to date, there is a widely accepted explanation for its experiential motivation (Núñez \& Cooperrider, 2013). Yet in the case of the future-uphill construal found in the Yupno valley and elsewhere, no obvious explanation presents itself. Here we consider several candidates.

Explanation 1: Entrance schema. In our initial report about the Yupno topographic system, we offered the following explanation for the future-uphill pattern: 
"The Yupno believe that their ancestors traveled up from an island offshore to settle in the Yupno valley. This may motivate an association between the ancestral past and the macro-scale downhill direction. More generally, the pattern may be motivated by an entrance schema. When inside the valley — or inside a traditional house - the entering action (from below) becomes metonymic for past times, and future as higher up is derived by contrast" (Núñez et al., 2012, p. 34).

This explanation is initially compelling in that it draws on a documented aspect of Yupno cosmology. It also accounts for the time gestures we observed indoors, in which participants generally construed the past as toward the door ('downhill,' according to the house's conceptual topography) and the future as away from the door ('uphill') (Núñez et al., 2012). Our enthusiasm for this explanation has since dimmed, however, for a few reasons. The first is that, as mentioned, other communities have since been documented that use the same mapping and no communities, to our knowledge, have yet been shown to use the opposite, future-downhill mapping. There are three other cases of future-uphill construals reported in the same region of Papua New Guinea (Pennington, 2016; Sarvasy, 2014; Taylor, 2015), as well as two off the coast (New Britain: Meng, 2018; Solomon Islands: Keesing, 1979); the same mapping is also found much further afield, in Tzeltal (Brown, 2012) and in languages of the Caucasus (Forker, 2020). Thus, any explanation of the Yupno pattern in terms of local cosmology and architectural practices might be an "undergeneralization" (Haspelmath, 1997) — that is, an explanation that is not general enough to accommodate the data. A second reason for doubt is that the ancestral origins explanation privileges a particular sense of downhill: the macroscale inclination of the valley, from the source of the Yupno river down toward the sea. But, as our examples here show, the Yupno time construal is much more flexible and adapted to local slopes. A last consideration is that it's not clear that an explanation of the future-uphill pattern needs to also account for time gestures indoors. It may be that the construal of time indoors simply piggybacks on the construal of space indoors. Again, when inside, one construes the area toward the door as downhill and 
away from the door as uphill (perhaps due to the mapping between house structure and valley structure; see Cooperrider, Slotta, \& Núñez, 2017, p. 791-3); past and future may then be construed according to this conceptual topography, with the past toward the door and the future away from it.

Explanation 2: Growth. Another possible explanation of the Yupno system is based on biological growth. Living things — whether human beings or bamboo stalks — tend to grow upward, so that there is, for at least some of the lifespan, a general trajectory of movement through time and movement upward. Part of the appeal of this explanation, beyond its intuitive plausibility, is that the use of growth as general model of time is well-documented in the ethnographic literature (Wiseman, 2019). The Yupno are subsistence horticulturalists and so there is little question that such a model would be culturally appropriate; bamboo in particular could be a compelling source due to its importance as a building material and its rapid, readily detectable growth. Growth also offers a general conceptual source, thus skirting the problem of undergeneralization; indeed, it has been suggested as the source of similar time mappings elsewhere (Forker, 2020, p. 14). However, there is at least one key weakness to the proposal: If the source for the Yupno time system were growth, the gestures we observed would likely look different. People almost invariably anchor their gestures about past and future to the actual terrain, not to some abstracted vertical axis. Many gestures are steeply pitched upward (e.g., Figure 3), but purely upward future gestures are extremely rare and purely downward past gestures are entirely absent. Moreover, the downward gestures we do observe are associated with 'now,' and it's not clear how these make sense within a growth model. It's certainly possible that the Yupno use growth as source for construing sequence time, such as when thinking about different life stages. Indeed, growth furnishes a natural conceptual source for thinking about 
sequence time but not a good one for thinking about deictic time. This is because it does not inherently involve an ego that is internal to the space itself, just an observer looking at a process from the outside (see Núñez \& Cooperrider, 2013) 5

Explanation 3: Looking downhill versus uphill. A third possibility concerns an intriguing parallel between the experience of viewing terrain and the experience of "viewing" time. When on sloped terrain, one can see more of the landscape when facing downhill than when facing uphill. (This is likely part of the reason for the general tendency to orient houses facing downhill.) Consider the case of a person walking up a mountain and occasionally stopping to view the terrain in all directions. When facing downhill, such an observer could often see a broad swath of terrain and possibly the specific path one approached by; and the higher up the observer went, the more they would be able to see when looking down. But, when facing uphill, it can be hard to see very much above and the path ahead may be obscured. These patterns are reminiscent of the human experience of time. It's easier to "see" what is in your past-as discussed in the Aymara case (Núñez \& Sweetser, 2006) — and the longer you go on, the more there is to see; the future, meanwhile can only be glimpsed vaguely if at all. This explanation has a number of virtues: it makes sense within the Yupno context; it fits the types of gestures we observed; and it provides a general explanation, one that would reasonably apply to any community engaged in daily travel over hilly terrain. However, admittedly this account does not have the intuitive simplicity of others, as it relies on an interaction of vision and topography.

\footnotetext{
${ }^{5}$ Note that, by this logic, graphical practices would seem to be a bad model for deictic time generally because they do not inherently involve the ego. This may be so, but literacy becomes a powerful model of deictic time nonetheless because of an additional practice of associating the ego's location with a position internal to the space itself (e.g., today's date in a calendar, or the ego's current "position" while reading text) (Núñez \& Cooperrider, 2013; see also Casasanto \& Jasmin, 2012).
} 
Explanation 4: Canonical travel. The experience of foot travel also motivates a fourth candidate explanation. This account is rooted in a common experience one has when navigating rugged terrain: it can feel like one spent the whole day walking uphill. A frequent pattern in the Yupno valley, for instance, is as follows: One leaves home early in the morning, spends much of the day elsewhere — walking between gardens and houses — and then returns home as evening sets in. On a day like this, in terms of pure elevation gain and loss, one will go uphill exactly as much as one went downhill. But because walking uphill is generally more effortful, one will have spent more time walking up than walking down. And, because of the extra exertion involved, walking uphill may also be more salient in memory. Put together, these facts may mean that walking uphill looms larger in memory and may thus become the "canonical" form of human travel. (Interestingly, uphill travel is described as the canonical form of travel in Tzeltal, though no rationale is offered [Brown \& Levinson, 1992, p. 597]). If canonical walking is indeed walking uphill for the Yupno, their construal of time might be considered a topographically inflected version of the future-in-front construal that is so widespread. Advantages of this account are that is relevant to the Yupno context but general enough to accommodate other cases; that it provides an intuitive model of deictic time, one that situates the ego within the array of events; and that it fits the gestures we observed. However, this account depends on a number of unknowns - in particular, whether walking uphill really is the canonical form of walking in Yupno. More fundamentally, the very notion of "canonical" forms of experience and how they shape construals requires further theoretical elaboration.

Each of the four explanations fulfills certain desiderata. The "looking downhill" and "canonical travel" explanations are general, compatible with gestures we observed, and relevant to the Yupno case. Of the two, the "canonical travel" explanation seems the more intuitive, due 
to its affinity with the cross-culturally widespread future-in-front construal. Meanwhile, the "entrance schema" explanation is the only one that simultaneously explains the outdoor and indoor time gestures we observed. It remains unclear whether this is truly an advantage, however, as the Yupno construal of time indoors may simply piggyback on the construal of space indoors. There are yet further explanations that have been suggested to us that might also be considered (see also Forker, 2020); and the three of us do not necessarily agree on which of the above explanations is most compelling. But by outlining and comparing the best explanations, we hope to spur future empirical work on future-uphill mappings, as well as further theoretical discussion of the experiential motivations for spatial construals of time more generally.

\section{Conclusion}

For more than half a century, linguists, anthropologists, philosophers, and psychologists have grappled with the puzzle of how humans make sense of time. Such research has documented widespread patterns, while also unearthing unexpected diversity (Núñez \& Cooperrider, 2013). Diversity in human concepts is of inherent humanistic interest, as part of the rich tapestry of practices, lifeways, and forms of sense-making our species has developed in different ecologies around the globe. It also has immeasurable scientific value. Often it is only by puzzling over the forces that generate apparent outliers that we come to understand more general patterns (e.g., Chrisomalis, 2020). We see environment-based understandings of time in just this light. Such systems may seem like marginal peculiarities, but, in fact, they stand to illuminate the major forces shaping human language, culture, and conceptualization. 
Unfortunately, the window to conduct such work appears to be closing (e.g., Cooperrider, 2019; Evans, 2010). The Yupno time system—given its dependence on a fragile ecology of practices and conditions - is undoubtedly precarious. And it's likely that any similar systems remaining in other parts of the world are also in jeopardy. The study of environment-based construals of time — and environment-based systems for making sense of the world, more generally_is thus not just humanistically and scientifically valuable. It's also, we would argue, urgent. 


\section{References}

Alibali, M. W. (2005). Gesture in spatial Cognition: Expressing, communicating, and thinking about spatial information. Spatial Cognition \& Computation, 5(4), 307-331.

Alverson, H. (1994). Semantics and experience: Universal metaphors of time in English, Mandarin, Hindi, and Sesotho. Johns Hopkins University Press.

Bender, A., \& Beller, S. (2014). Mapping spatial frames of reference onto time: A review of theoretical accounts and empirical findings. Cognition, 132(3), 342-382.

https://doi.org/10.1016/j.cognition.2014.03.016

Bergen, B. K., \& Lau, T. T. C. (2012). Writing direction affects how people map space onto time. Frontiers in Psychology, 3(April), 1-5. https://doi.org/10.3389/fpsyg.2012.00109

Bickel, B. (1997). Spatial operations in deixis, cognition, and culture: Where to orient oneself in Belhare. In J. Nuyts \& E. Pederson (Eds.), Language and Conceptualization (pp. 46-83). Cambridge University Press.

Bohnemeyer, J. (2009). Temporal anaphora in a tenseless language. In W. Klein \& P. Li (Eds.), The expression of time in language (pp. 83-128). Mouton de Gruyter.

Boroditsky, L. (2000). Metaphoric structuring: Understanding time through spatial metaphors. Cognition, 75(1), 1-28.

Boroditsky, L., \& Gaby, A. (2010). Remembrances of times east: Absolute spatial representations of time in an Australian Aboriginal community. Psychological Science, 21(11), 1635-1639. https://doi.org/10.1177/0956797610386621

Bowern, C. (2012). A grammar of Bardi (Vol. 57). Walter de Gruyter.

Brown, P. (2012). Time and space in Tzeltal: Is the future uphill? Frontiers in Psychology, 3(July). https://doi.org/10.3389/fpsyg.2012.00212 
Brown, P., \& Levinson, S. C. (1992). "Left" and "right" in Tenejapa: Investigating a linguistic and conceptual gap. Zeitschrift Für Phonetik, Sprachwissenschaft Und Kommunikationsforschung, 45(6), 590-611.

Brown, P., \& Levinson, S. C. (1993). “Uphill” and “downhill” in Tzeltal. Journal of Linguistic Anthropology, 3(1), 46-74. https://doi.org/10.1525/jlin.1993.3.1.46

Casasanto, D., \& Boroditsky, L. (2008). Time in the mind: Using space to think about time. Cognition, 106(2), 579-593. https://doi.org/10.1016/j.cognition.2007.03.004

Casasanto, D., \& Jasmin, K. (2012). The hands of time: Temporal gesture in English speakers. Cognitive Linguistics, 23(4), 643-674.

Chrisomalis, S. (2020). Reckonings: Numerals, cognition, and history. MIT Press.

Cienki, A., \& Müller, C. (Eds.). (2008). Metaphor and gesture. John Benjamins Publishing.

Clark, H. H. (1973). Space, time, semantics, and the child. In T. E. Moore (Ed.), Cognitive development and the acquisition of language (pp. 28-63). Academic Press.

Cooperrider, K. (2017). Foreground gesture, background gesture. Gesture, 16(3), 176-202. https://doi.org/10.1075/gest.16.2.02coo

Cooperrider, K. (2019, January 23). What happens to cognitive diversity when everyone is more WEIRD? Aeon. https://aeon.co/ideas/what-happens-to-cognitive-diversity-when-everyone-ismore-weird

Cooperrider, K., Marghetis, T., \& Núñez, R. (2017). Where does the ordered line come from? Evidence from a culture of Papua New Guinea. Psychological Science, 28(5), 599-608. https://doi.org/10.1177/0956797617691548

Cooperrider, K., \& Núñez, R. (2009). Across time, across the body: Transversal temporal gestures. Gesture, 9(2), 181-2006. https://doi.org/10.1075/gest.9.2.02coo 
Cooperrider, K., \& Núñez, R. (2012). Nose-pointing: Notes on a facial gesture of Papua New Guinea. Gesture, 12(2), 103-129. https://doi.org/10.1075/gest.12.2.01coo

Cooperrider, K., Slotta, J., \& Núñez, R. (2017). Uphill and downhill in a flat world: The conceptual topography of the Yupno house. Cognitive Science, 41, 768-799. https://doi.org/10.1111/cogs. 12357

Cooperrider, K., Slotta, J., \& Núñez, R. (2018). The preference for pointing with the hand is not universal. Cognitive Science, 1-16. https://doi.org/10.1111/cogs.12585

Danziger, E. (2011). Distinguishing three-dimensional forms from their mirror-images: Whorfian results from users of intrinsic frames of linguistic reference. Language Sciences, 33(6), 853867. https://doi.org/10.1016/j.langsci.2011.06.008

De León, L. (1994). Exploration in the acquisition of geocentric location by Tzotzil children. Linguistics, 32(4-5). https://doi.org/10.1515/ling.1994.32.4-5.857

Evans, N. (2010). Dying words: Endangered languages and what they have to tell us. WileyBlackwell.

Fedden, S., \& Boroditsky, L. (2012). Spatialization of time in Mian. Frontiers in Psychology, 3(November), 1-9. https://doi.org/10.3389/fpsyg.2012.00485

Forker, D. (2019). Elevation as a category of grammar: Sanzhi Dargwa and beyond. Linguistic Typology, 23(1), 59-106. https://doi.org/10.1515/lingty-2019-0001

Forker, D. (2020). Elevation as a grammatical and semantic category of demonstratives. Frontiers in Psychology, 11, 1712. https://doi.org/10.3389/fpsyg.2020.01712 François, A. (2004). Reconstructing the geocentric system of Proto-Oceanic. Oceanic Linguistics, 43(1), 1-31. https://doi.org/10.1353/ol.2004.0009 
Fuhrman, O., \& Boroditsky, L. (2010). Cross-cultural differences in mental representations of time: Evidence from an implicit nonlinguistic task. Cognitive Science, 34(8), 1430-1451. https://doi.org/10.1111/j.1551-6709.2010.01105.x

Gaby, A. (2012). The Thaayorre think of time like they talk of space. Frontiers in Psychology, 3(August), 1-8. https://doi.org/10.3389/fpsyg.2012.00300

Gorenflo, L. J., Romaine, S., Mittermeier, R. A., \& Walker-Painemilla, K. (2012). Cooccurrence of linguistic and biological diversity in biodiversity hotspots and high biodiversity wilderness areas. PNAS, 109(21), 8032-8037. https://doi.org/10.1073/pnas.1117511109

Gu, Y., Zheng, Y., \& Swerts, M. (2019). Which is in front of Chinese people, past or future? The effect of language and culture on temporal gestures and spatial conceptions of time. Cognitive Science, 43(12). https://doi.org/10.1111/cogs.12804

Haspelmath, M. (1997). Indefinite pronouns. Oxford University Press.

Haun, D. B. M., Rapold, C. J., Janzen, G., \& Levinson, S. C. (2011). Plasticity of human spatial cognition: Spatial language and cognition covary across cultures. Cognition, 119, 70-80. https://doi.org/10.1016/j.cognition.2010.12.009

Haviland, J. B. (1998). Guugu Yimithirr cardinal directions. Ethos, 26(1), 25-47.

Hoffmann, D. (2019). Restrictions on the usage of spatial frames of reference in location and orientation descriptions: Evidence from three Australian languages. Australian Journal of Linguistics, 39(1), 1-31. https://doi.org/10.1080/07268602.2019.1542927

Hutchins, E. (2010). Cognitive ecology. Topics in Cognitive Science, 2(4), 705-715. https://doi.org/10.1111/j.1756-8765.2010.01089.x 
Jurafsky, D. (1996). Universal tendencies in the semantics of the diminutive. Language, 72(3), 533-578.

Keck, V. (2005). Social discord and bodily disorders: Healing among the Yupno of Papua New Guinea. Carolina Academic Press.

Keesing, R. (1979). Linguistic knowledge and cultural knowledge: Some doubts and speculations. American Anthropologist, 81(1), 14-36.

Kendon, A. (2004). Gesture: Visible action as utterance. Cambridge University Press.

Kita, S. (2009). Cross-cultural variation of speech-accompanying gesture: A review. Language and Cognitive Processes, 24(2), 145-167. https://doi.org/10.1080/01690960802586188

Lakoff, G., \& Johnson, M. (1980). Metaphors we live by. University of Chicago Press.

Le Guen, O. (2011). Speech and gesture in spatial language and cognition among the Yucatec Mayas. Cognitive Science, 35, 905-938. https://doi.org/10.1111/j.1551-6709.2011.01183.x

Le Guen, O., \& Balam, L. I. P. (2012). No metaphorical timeline in gesture and cognition among Yucatec Mayas. Frontiers in Psychology, 3(August), 1-15.

https://doi.org/10.3389/fpsyg.2012.00271

Levinson, S. C., \& Brown, P. (1994). Immanuel Kant among the Tenejapans: Anthropology as empirical philosophy. Ethos, 22(1), 3-41.

Levinson, S. C., \& Wilkins, D. (2006). Grammars of space. Cambridge University Press.

Li, P., \& Gleitman, L. (2002). Turning the tables: Language and spatial reasoning. Cognition, $83(3), 265-294$.

Marghetis, T., McComsey, M., \& Cooperrider, K. (2020). Space in hand and mind: Gesture and spatial frames of reference in bilingual Mexico. Cognitive Science, 44(12).

https://doi.org/10.1111/cogs.12920 
McTaggart, J. (1908). The unreality of time. Mind, 17, 457-474.

Meng, C. (2018). A Grammar of Tulil [Doctoral Dissertation, La Trobe University]. La Trobe University Repository. http://hdl.handle.net/1959.9/565684

Moore, K. E. (2014). The spatial language of time. John Benjamins.

Núñez, R., \& Cooperrider, K. (2013). The tangle of space and time in human cognition. Trends in Cognitive Sciences, 17(5), 220-229. https://doi.org/10.1016/j.tics.2013.03.008

Núñez, R., Cooperrider, K., Doan, D., \& Wassmann, J. (2012). Contours of time: Topographic construals of past, present, and future in the Yupno valley of Papua New Guinea. Cognition, 124(1), 25-35. https://doi.org/10.1016/j.cognition.2012.03.007

Núñez, R., \& Cornejo, C. (2012). Facing the sunrise: Cultural worldview underlying intrinsicbased encoding of absolute frames of reference in Aymara. Cognitive Science, 36(6), 965991. https://doi.org/10.1111/j.1551-6709.2012.01237.x

Núñez, R., \& Sweetser, E. (2006). With the future behind them: Convergent evidence from Aymara language and gesture in the crosslinguistic comparison of spatial construals of time. Cognitive Science, 30(3), 401-450. https://doi.org/10.1207/s15516709cog0000_62

Palmer, B. (Ed.). (2017). The languages and linguistics of the New Guinea area: A comprehensive guide. Walter de Gruyter.

Palmer, B., Lum, J., Schlossberg, J., \& Gaby, A. (2017). How does the environment shape spatial language? Evidence for sociotopography. Linguistic Typology, 21(3), 457-491. https://doi.org/10.1515/lingty-2017-0011

Pederson, E., Danziger, E., Wilkins, D., Levinson, S. C., Kita, S., \& Senft, G. (1998). Semantic Typology and Spatial Conceptualization. Language, 74(3), 557-589. 
Pennington, R. (2016). A grammar of Ma Manda: A Papuan Language of Morobe Province, Papua New Guinea [Doctoral Dissertation, James Cook University]. James Cook University repository. https://researchonline.jcu.edu.au/48926/

Post, M. W. (2019). Topographical deixis in Trans-Himalayan (Sino-Tibetan) languages. Transactions of the Philological Society, 00, 1-22. https://doi.org/10.1111/1467-968X.12155

Rinaldi, L., Locati, F., Parolin, L., Bernardi, N. F., \& Girelli, L. (2016). Walking on a mental time line: Temporal processing affects step movements along the sagittal space. Cortex, 4-7. https://doi.org/10.1016/j.cortex.2016.02.013

Saint Augustine. (2008). Confessions (Garry Wills, Trans.). Penguin. (Original work published ca. 400 C.E.)

Santiago, J., Lupiáñez, J., Pérez, E., \& Funes, M. J. (2007). Time (also) flies from left to right. Psychonomic Bulletin \& Review, 14(3), 512-516.

Sarvasy, H. S. (2014). A grammar of Nungon: A Papuan language of the Morobe Province, Papua New Guinea [Doctoral Dissertation, James Cook University]. James Cook University repository. https://researchonline.jcu.edu.au/40832/

Schlossberg, J. (2018). Atolls, islands and endless suburbia: Spatial reference in Marshallese [Doctoral Dissertation]. The University of Newcastle.

Schmidt, C. K. (1991). Of people and plants: A botanical ethnography of Nokopo Village, Madang and Morobe Provinces, Papua New Guinea. Basler Beiträge zur Ethnologie.

Senft, G. (Ed.) (1997). Referring to space: Studies in Austronesian and Papuan languages. Oxford University Press. 
Shapero, J. A. (2016). Does environmental experience shape spatial cognition? Frames of reference among Ancash Quechua Speakers (Peru). Cognitive Science, 1-25. https://doi.org/10.1111/cogs. 12458

Slotta, J. (2012). Dialect, trope, and enregisterment in a Melanesian speech community. Language \& Communication, 32(1), 1-13. https://doi.org/10.1016/j.langcom.2011.11.003

Slotta, J. (2014). Sketch Grammar. Documenting Yopno Diversity: Dialect Variation in a Papuan Language. Endangered Languages Archive. https://www.elararchive.org/uncategorized/SO_48699f22-2bb2-4cea-be88-017c805ac781/. Accessed on September 27, 2021.

Slotta, J. (2015). The perlocutionary is political: Listening as self-determination in a Papua New Guinean polity. Language in Society, 44(4), 525-552. https://doi.org/10.1017/S0047404515000421

Soto, V. V. (2011). The "uphill” and "downhill” system in Meseño Cora. Language Sciences, 33(6), 981-1005. https://doi.org/10.1016/j.langsci.2011.06.011

Starr, A., \& Srinivasan, M. (2021). The future is in front, to the right, or below: Development of spatial representations of time in three dimensions. Cognition, 210, 104603. https://doi.org/10.1016/j.cognition.2021.104603

Taylor, M. A. (2015). Nukna grammar sketch. SIL-PNG.

Tillman, K. A., Marghetis, T., Barner, D., \& Srinivasan, M. (2017). Today is tomorrow's yesterday: Children's acquisition of deictic time words. Cognitive Psychology, 92, 87-100. https://doi.org/10.1016/j.cogpsych.2016.10.003 
Traugott, E. C. (1978). On the expression of spatio-temporal relations in language. In J. H.

Greenberg (Ed.), Universals of Human Language. Volume 3: Word Structure (pp. 369-400).

Stanford University Press.

Urban, M. (2020). Mountain linguistics. Language and Linguistics Compass, 14(9).

https://doi.org/10.1111/lnc3.12393

Valenzuela, J., Cánovas, C. P., Olza, I., \& Carrión, D. A. (2020). Gesturing in the wild: Evidence for a flexible mental timeline. Review of Cognitive Linguistics, 18(2), 289-315.

Von Heeschen, V. (1982). Some systems of spatial deixis in Papuan languages. In J.

Weissenborn \& W. Klein (Eds.), Here and There: Cross-linguistic Studies on Deixis and Demonstration (pp. 81-109). John Benjamins.

Walker, E., Bergen, B. K., \& Núñez, R. (2017). The spatial alignment of time: Differences in alignment of deictic and sequence time along the sagittal and lateral axes. Acta Psychologica, $175,13-20$.

Walker, E., \& Cooperrider, K. (2016). The continuity of metaphor: Evidence from temporal gestures. Cognitive Science, 40, 481-495. https://doi.org/10.1111/cogs.12254

Wassmann, J. (1993). Worlds in mind: The experience of an outside world in a community of the Finisterre Range of Papua New Guinea. Oceania, 64(2), 117-145.

Wassmann, J. (1994). The Yupno as post-Newtonian Scientists: The question of what is "natural" in spatial description. Man, 29(3), 645-666.

Wassmann, J. (2016). The gently bowing person: An ideal among the Yupno in Papua New Guinea. Universitatsverlag Winter. 
Wassmann, J., \& Dasen, P. R. (1998). Balinese spatial orientation: Some empirical evidence of moderate linguistic relativity. The Journal of the Royal Anthropological Institute, 4(4), 689711.

Whorf, B. L. (1956). The relation of habitual thought and behavior to language. In J. Carroll (Ed.), Language, Thought, and Reality (pp. 134-159). MIT Press.

Wiseman, R. (2019). Getting beyond rites of passage in archaeology: Conceptual metaphors of journeys and growth. Current Anthropology, 60(4), 449-474. https://doi.org/10.1086/704696 